\title{
Insights into brain microstructure from in vivo DW-MRS
}

\author{
Marco Palombo $^{\mathrm{a}, *}$, Noam Shemesh ${ }^{\mathrm{b}}$, Itamar Ronen ${ }^{\mathrm{c}}$, Julien Valette ${ }^{\mathrm{d}, \mathrm{e}}$ \\ a Department of Computer Science, Centre for Medical Image Computing, University College of London, Gower Street, London WC1E 6BT, United Kingdom \\ ${ }^{\mathrm{b}}$ Champalimaud Neuroscience Programme, Champalimaud Centre for the Unknown, Av. Brasilia, 1400-038 Lisbon, Portugal \\ ${ }^{\text {c }}$ C. J. Gorter Center for High Field MRI, Department of Radiology, Leiden University Medical Center, Leiden 2333ZA, The Netherlands \\ d Commissariat à l'Energie Atomique et aux Energies Alternatives (CEA), Direction de la Recherche Fondamentale (DRF), Institut de Biologie François Jacob, MIRCen, F- \\ 92260 Fontenay-aux-Roses, France \\ ${ }^{\mathrm{e}}$ Centre National de la Recherche Scientifique (CNRS), Université Paris-Sud, Université Paris-Saclay, UMR 9199, Neurodegenerative Diseases Laboratory, F-92260 \\ Fontenay-aux-Roses, France
}

\section{A R T I C L E I N F O}

\section{Keywords:}

Diffusion

Metabolites

Intracellular space

Cell structure

Tissue microstructure

Brain

${ }^{1} \mathrm{H}$ magnetic resonance spectroscopy

\begin{abstract}
A B S T R A C T
Many developmental processes, such as plasticity and aging, or pathological processes such as neurological diseases are characterized by modulations of specific cellular types and their microstructures. Diffusion-weighted Magnetic Resonance Imaging (DW-MRI) is a powerful technique for probing microstructure, yet its information arises from the ubiquitous, non-specific water signal. By contrast, diffusion-weighted Magnetic Resonance Spectroscopy (DW-MRS) allows specific characterizations of tissues such as brain and muscle in vivo by quantifying the diffusion properties of MR-observable metabolites. Many brain metabolites are predominantly intracellular, and some of them are preferentially localized in specific brain cell populations, e.g., neurons and glia. Given the microstructural sensitivity of diffusion-encoding filters, investigation of metabolite diffusion properties using DW-MRS can thus provide exclusive cell and compartment-specific information. Furthermore, since many models and assumptions are used for quantification of water diffusion, metabolite diffusion may serve to generate a-priori information for model selection in DW-MRI. However, DW-MRS measurements are extremely challenging, from the acquisition to the accurate and correct analysis and quantification stages. In this review, we survey the state-of-the-art methods that have been developed for the robust acquisition, quantification and analysis of DWMRS data and discuss the potential relevance of DW-MRS for elucidating brain microstructure in vivo. The review highlights that when accurate data on the diffusion of multiple metabolites is combined with accurate computational and geometrical modeling, DW-MRS can provide unique cell-specific information on the intracellular structure of brain tissue, in health and disease, which could serve as incentives for further application in vivo in human research and clinical MRI.
\end{abstract}

\section{Introduction}

Nuclear Magnetic Resonance (NMR) signals are incredibly rich sources of information because they are sensitive to a multitude of physical mechanisms, each capable of portraying different physicochemical aspects of the spin ensembles. The chemical shift - the slight, ppm-scale variations in NMR frequency due to electronic interactions in a given molecule - was perhaps one of the earliest NMR interactions to be discovered, and its utilization has transformed Chemistry due to its ability to characterize molecular structure, first in small molecules and then in much bigger molecules such as proteins and membranes. It is therefore not a surprise that NMR's spatially-localized version - rebranded Magnetic Resonance Spectroscopy (MRS) - has had a strong impact on biomedicine and neuroscience. The spectral dimension revealed by MRS in general, and by ${ }^{1} \mathrm{H}$ MRS in particular, allows a unique way to characterize tissues in vivo by quantifying the metabolite levels, at least for those that exist at relatively high concentrations (typically $>1 \mathrm{mM}$ ). In high-quality MRS spectra, about 20 metabolites have been identified and quantified in vivo, including neurotransmitters, energy-related metabolites, osmolytes and others (Tkac et al., 2009). Accurate information on metabolite concentrations in tissue found vast applications: to name but a few, MRS has been used for characterization of energy-cycle metabolism in vivo (Cerdan et al., 1990; Kunnecke et al., 1993; Duarte et al., 2012), identifying and differentiating between tumor types (Lee and Gonzalez, 2000) and gaining insights into neurotransmitter turnover upon activation (Rothman et al., 1999). Such studies have been

\footnotetext{
* Corresponding author.

E-mail address: mrc.palombo@gmail.com (M. Palombo).
} 
instrumental for both clinical applications as well as for basic science.

Diffusion is another mechanism that has made a huge impact in biomedicine, neuroscience and other fields. The spins giving rise to sharp NMR signals are never stationary, and in the presence of magnetic field gradients - be they internal, i.e. caused by tissue susceptibility variations (Hahn, 1950), or externally applied (Stejskal and Tanner, 1965) - the NMR signal will also reflect information on the diffusion process that the ensemble had undergone. In solution, the signal attenuation in the presence of magnetic field gradients reflects the diffusion coefficient, which have been used for example, as a second dimension for resolving the constituents in chemical mixtures in which chemical shift spectral lines overlap (Morris and Johnson, 1993). When diffusion is restricted by the presence of physical barriers, the diffusion signal becomes imprinted with signatures of the confining geometry, and hence its measurement may be capable of reporting on microstructure. Fortuitously, the typical time-scales of diffusion encodings are compatible with the time required for water molecules to traverse (sub)cellular length scales at $37^{\circ} \mathrm{C}$. Thus, water diffusion is sensitive to structures on a spatial scale much smaller than the voxel size, an advantage that found applications abound, for example, in early detection of stroke (Vangelderen et al., 1994; Moseley et al., 1990; Schlaug et al., 1997) as well as in fiber orientation mapping and tracking (Mori and van Zijl, 2002; Lori et al., 2002) as well as other applications in biomedicine (Le Bihan et al., 2001).

Why then would we go beyond quantifying microstructures from water signals? Water is present in almost every microscopic as well as macroscopic tissue subcomponent, and is thus inherently non-specific. Water in blood vessels, CSF and cysts diffuses with a very high diffusion coefficient, and flow is also present. In neural tissue, all cells, including astrocytes, neurons, oligodendrocytes or other glia, contain water; furthermore, their subcellular units (cell body, neurites/astrocytic processes, cell mitochondria or cytoplasm, etc.) are also very similar in water content. Different cell types such as excitatory/inhibitory/modulatory neurons, are also not different from the water content perspective. In addition to water in cells, it is important to note also the extracellular space, which also contains water protons at similar concentration $(\sim 110 \mathrm{M})$ as the intracellular environment. Since the exact diffusion coefficients or properties in all cellular, subcellular, and extracellular compartments are still widely unknown (Lampinen et al., 2017; Kiselev, 2017), it is extremely difficult to gain compartment-specific information from water-based measurements.

By contrast with water, most metabolites are predominantly intracellular, and some metabolites are even cell-specific; that is, they are produced by or confined to a specific cellular population (Fig. 1). Perhaps the most obvious example is N-Acetylaspartate (NAA): numerous studies (Gill et al., 1989; Simmons et al., 1991; Petroff et al., 1993) have found that in the nervous system, the osmolyte NAA is produced mainly by neurons, and furthermore, NAA is not secreted by neurons. A very recent study evidenced the production of NAA by oligodendrocytes and confinement of that NAA pool in the myelin that unsheathes the neuronal axons (Nordengen et al., 2015). Hence, NAA is considered specific to the neuronal (or neuronal-associated) intracellular compartment. Luckily, NAA is also one of the most quantifiable metabolites, having both very high concentration $(\sim 20 \mathrm{mM})$, higher than all other metabolites other than glutamate (Glu), and a very convenient singlet at the $2.02 \mathrm{ppm}$ resonance, which is not modulated over different TEs. In some cases, there are some modulated metabolite signals that can "hide" under NAA's resonance, especially at lower fields and/or when lines are somewhat broad (e.g. a 1.95 ppm GABA methine quintet).

Another metabolite with high compartmental specificity is myoinositol (Ins). In the CNS, Ins has been shown to be predominantly localized in astrocytes (Gill et al., 1989; Choi et al., 2007; Brand et al., 1993). This provides, in principle, a very elegant counterpart to NAA's neuronal preponderance, as Ins represents the complementary major intracellular compartment in the CNS. Other metabolites, such as the co-
Tissue Composition
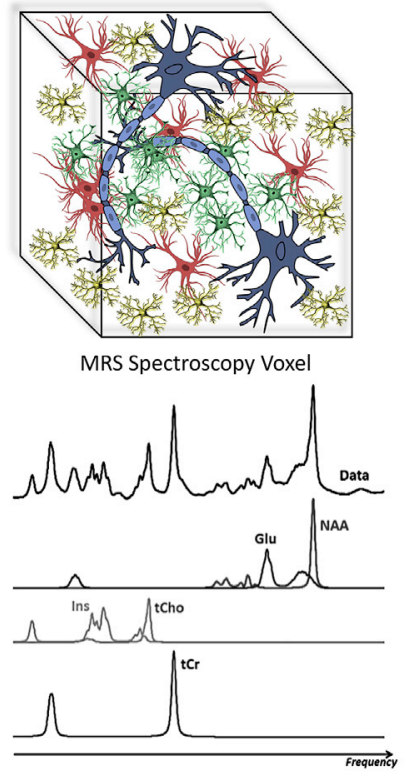

Metabolites Compartmentation

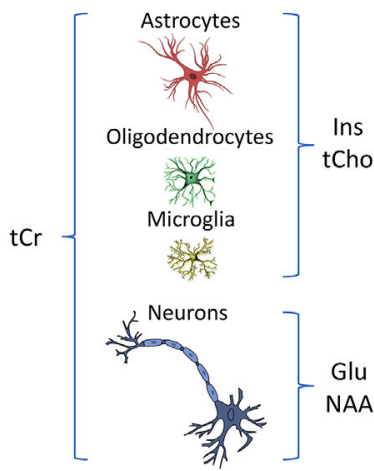

Fig. 1. Schematic representation of the composition of a spectroscopic voxel within the brain tissue, together with the generally accepted (although still debated) cell-specific compartmentalization of brain metabolites. Typical MRS spectra and the specific peaks associated to the most investigated metabolites (NAA, Glu, tCr, tCho and Ins) are also schematized.

measured pair creatine and phosphocreatine (tCr) (Molloy et al., 1992; Beard and Braissant, 2010) and the co-measured choline compounds (tCho), have been shown to originate predominantly from Glia (up to $\sim 75 \%$ specificity for tCho) (Brand et al., 1993). Le Belle et al. (2002) reported tCho concentration of $1.5-2.2 \mu \mathrm{mol} / \mathrm{g}$ in brain tissue using two different extraction techniques, and $27-41 \mu \mathrm{mol} / \mathrm{g}$ in astrocytes. When normalized to $\mathrm{tCr}$, this corresponds to $[\mathrm{tCho}] /[\mathrm{tCr}]=0.15-0.25$ in brain tissue (close to what is indeed typically measured in the brain in vivo (Duarte et al., 2012)), and [tCho]/[tCr] $=1.9-2.4$ in astrocytes. This represents at least a 10 times higher concentration in astrocytes than in neurons. Though maybe less-specific than the Ins counterpart, the signal of $\mathrm{tCr}$ and tCho is readily quantifiable as both represent uncoupled N-bound methyl singlets.

The motivation for quantifying diffusion properties of these metabolites is now perhaps clearer: their diffusion properties may reflect specific cell-type geometry. Since many diseases or disease phases are characterized by injury to specific cellular types (e.g., glioblastoma affecting glial cells) or compartments, and since water signals are not necessarily representative of any specific compartment, metabolite signals can serve as biomarkers with enhanced specificity. Additionally, information on how metabolites such as neurotransmitters change compartments (e.g., upon neurotransmission) could complement fMRI with a more direct observation of neural activation in vivo (Mangia et al., 2009; Branzoli et al., 2013). Furthermore, since many models and assumptions are used for quantification of water diffusion, metabolite diffusion may serve to generate a-priori information for model selection.

The purpose of this review is to introduce the potential relevance of diffusion-weighted MRS for elucidating brain microstructure in vivo. For a more exhaustive survey of MRS or diffusion at large, the reader is referred to the following exhaustive reviews of diffusion-weighted MRS (Nicolay et al., 2001; Cao and Wu, 2017; Ronen and Valette, 2015), and DW-MRI in general (Kiselev, 2017; Basser, 1995; Basser and Jones, 2002; Grebenkov, 2007; Yablonskiy and Sukstanskii, 2010).

For this review, the reader is assumed to be familiar with general definitions and notations of molecular diffusion and NMR diffusion 
measurements, and in particular with DW-MRI. The review is structured as follows: in Section 1, methodological aspects of data acquisition and quantification in DW-MRS are introduced and compared to DW-MRI; in Section 2, the most recent models proposed to describe diffusion of brain metabolites in the intra-cellular space are reviewed, together with some examples of recent successful applications to experimental data based on advanced diffusion encoding techniques; In Section 3, we briefly discuss co-analysis of DW-MRI and DW-MRS data: what has been done so far and what are some of the possible directions in which this approach can evolve to provide a more comprehensive picture of tissue microstructure.

\section{DW-MRS methodology}

In this section, we present methodological aspects of data acquisition and quantification specific to DW-MRS, as compared to DW-MRI or nondiffusion weighted MRS. For more exhaustive reviews of DW-MRS basic methods and applications, the reader is referred to (Nicolay et al., 2001; Cao and Wu, 2017; Ronen and Valette, 2015).

Sometimes, NMR jargon is used. For those readers who are not sufficiently familiar with that jargon, we refer to (Callaghan, 1991; Keeler, 2011) for help.

\section{A. DW-STEAM sequence}

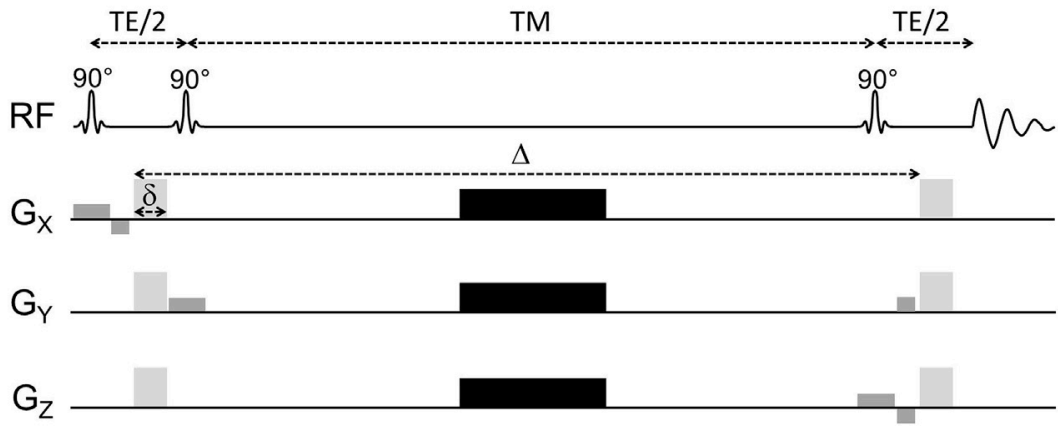

\section{B. Effect of cross-terms in DW-STEAM}

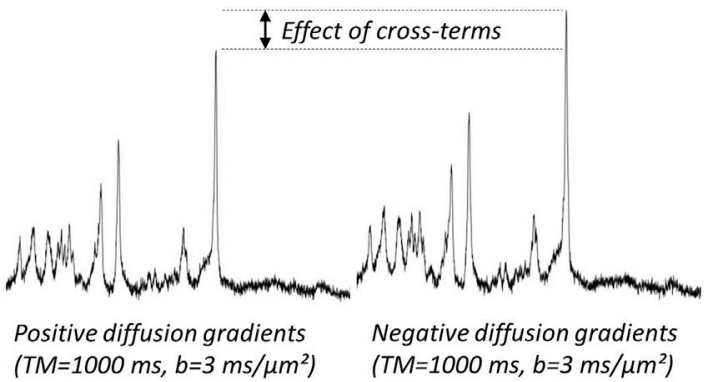

\section{Bipolar DW-PRESS sequence}

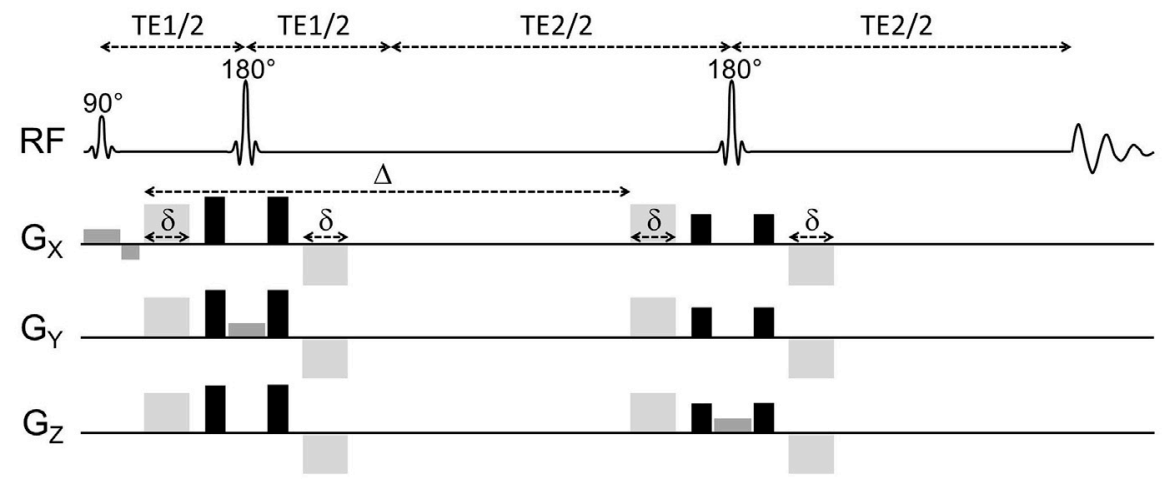

\section{STE-LASER sequence}

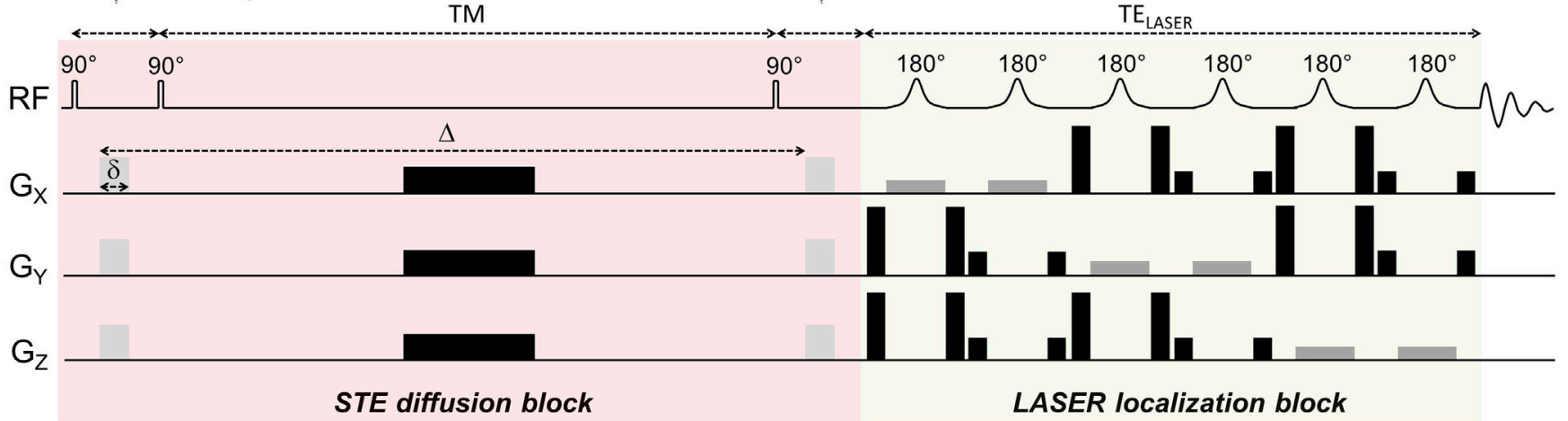

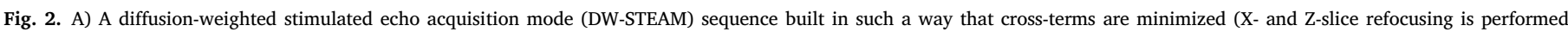

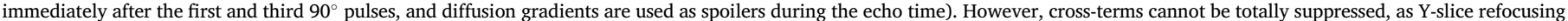

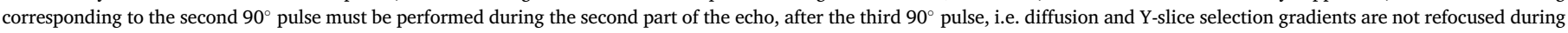

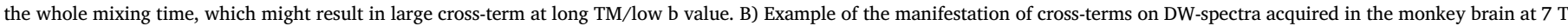

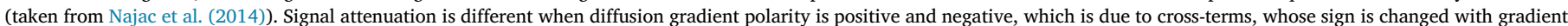

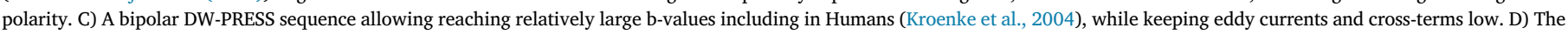

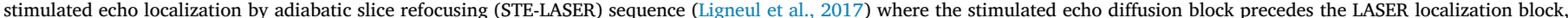

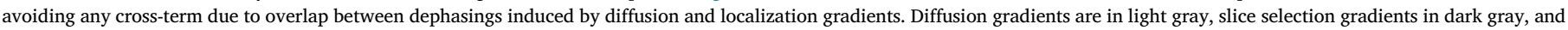
spoiler gradients in black. 
DW-MRS pulse sequences: specific constraints, traditional approaches, recent developments

In vivo DW-MRS pulse sequences must achieve several goals simultaneously: (1) adequate diffusion-weighting, (2) efficient and accurate signal localization to the volume of interest and (3) robust acquisition of free induction decay (FID) to obtain the chemical shift spectral dimension. This is usually achieved by implementing diffusion-sensitizing gradients within a conventional localized MRS sequence, such as STEAM (STimulated Echo Acquisition Mode) (Frahm et al., 1987), PRESS (Point RESolved Spectroscopy) (Bottomley, 1987) or LASER (Localization by Adiabatic SlicE Refocusing) (Garwood and DelaBarre, 2001).

In the early days of DW-MRS, because of the relatively low maximal gradient strength available on MRI scanners, the stimulated echo-based DW-STEAM pulse sequence (Moonen et al., 1990; Posse et al., 1993) was the most convenient way to reach sufficiently high b-values in vivo (typically 5-10 times stronger than that used for water, since metabolite apparent diffusion coefficient, or ADC, is about $0.1-0.2 \mu \mathrm{m}^{2} / \mathrm{ms}$ versus $0.5-1 \mu \mathrm{m}^{2} / \mathrm{ms}$ for water in tissue, suggesting that for the product bD to attenuate, larger b-values are required for metabolite signals (Ellegood et al., 2011)). Towards this goal, the diffusion time is built around the mixing time, which can be long without excessive signal loss due to relaxation (as during the mixing time magnetization relaxes according to $\mathrm{T}_{1}$, typically 1-2 s for metabolites (de Graaf et al., 2006; Shemesh et al., 2014a)): diffusion-sensitizing gradient lobes are inserted within the echo time, between the first and second slice-selective $90^{\circ} \mathrm{RF}$ pulses for dephasing, and after the third slice-selective $90^{\circ} \mathrm{RF}$ pulse for rephasing (see Fig. 2A). This is advantageous to reach high b-values but also to specifically study diffusion at long diffusion times. In addition, because of the short echo time that can be reached, STEAM allows quantifying the signal of more heavily J-modulated metabolites such as Glu or Ins. Another noteworthy feature of STEAM is its generally excellent performance in terms of water suppression, because an additional water suppression RF pulse can be inserted during the mixing time. However, one issue with STEAM is that the cross-terms with other gradients tend to be very large, as shown in Fig. 2B. This is because cross-terms build up during the mixing time, which can be quite long in STEAM, and furthermore, the cross-terms are proportional to the cube of time, making these effects potentially highly significant. It is therefore critical to pay extra attention to cross-terms, either by calculating them, or minimizing their effect by performing two successive acquisitions with opposite diffusion gradient polarities and calculating the geometric average of signal attenuations measured with both polarities (Jara and Wehrli, 1994; Neeman et al., 1991; Najac et al., 2014; Shemesh and Cohen, 2011). The most significant drawback with stimulated echo results is certainly the loss of half the magnetization. Therefore, a spin echo sequence might be preferred when experimentally possible, as sensitivity is one of the major issues of MRS in general, and of DW-MRS in particular.

The first spin echo DW-MRS sequences were based on PRESS, and performed on preclinical systems as gradient strength was enough to reach sufficient b-values (van der Toorn et al., 1996; Dijkhuizen et al., 1999). Identical pairs of gradient pulses $G$ were inserted around one or the two slice-selective $180^{\circ}$ RF pulses ( $90^{\circ}$; G; $180^{\circ}$; G; G; $180^{\circ}$; G), which allowed compensating for phase variation due to bulk translational motion, provided that motion remains constant during the sequence. The sequence was subsequently modified for clinical implementation by applying a bipolar gradient scheme (Kroenke et al., 2004) $\left(90^{\circ} ; \mathrm{G} ; 180^{\circ}\right.$; -G; G; $180^{\circ}$; -G) (Fig. 2C), thus taking advantage of the twice-refocused spin echo to increase maximal achievable diffusion-weighting, while minimizing eddy currents and mitigating, to a certain extent, cross-terms with time-constant background gradients (Shemesh and Cohen, 2011; Zheng and Price, 2007). A DW-PRESS sequence with specific gradient scheme has also been proposed to provide single-shot isotropic diffusion-weighting (i.e. weighting by the diffusion tensor) (de Graaf et al., 2001), which may be of interest if one is only interested in rotationally invariant indexes, e.g. the mean diffusivity, in highly anisotropic voxels such as in white matter bundles.

Another type of double spin-echo sequence incorporating fully adiabatic refocusing pulses, termed LASER (Garwood and DelaBarre, 2001), has been gaining increasing attention. LASER offers superior localization performance compared to STEAM or PRESS, as well as insensitivity to inhomogeneities of the radiofrequency making LASER a particularly attractive localization scheme, also for diffusion-weighted MRS as pioneered in (Kroenke et al., 2003). In one variant, oscillating gradients were inserted around the first $180^{\circ}$ refocusing pulse to measure metabolite diffusion at very short time scales (Marchadour et al., 2012; Ligneul and Valette, 2017). In another variant, three pairs of gradient pulses of opposite polarities were inserted around the three pairs of refocusing pulses to achieve single-shot isotropic diffusion-weighting while minimizing cross-terms with other gradients (Valette et al., 2012). In addition to previously mentioned advantages, the six successive refocusing pulses in LASER make it a CPMG sequence, resulting in slightly longer $\mathrm{T}_{2}$ and reduced J-modulation compared to PRESS, which is beneficial for the detection of Glu and Ins. Unfortunately, LASER may be difficult to perform in Humans at high fields $(\geq 7 \mathrm{~T}$ ) because of specific absorption rate (SAR) issues, but more practical variants such as semi-LASER, in which the excitation pulse is a non-adiabatic selective pulse, may be employed.

A recent development in MRS sequence design consists in temporally isolating diffusion-weighting from localization. So far, this strategy has been implemented on preclinical systems by successively playing out three blocks: non-spatially-selective excitation, diffusion-weighting, and LASER localization (Shemesh et al., 2014b; Ligneul et al., 2017), as shown in Figs. 2D and 3. The main advantages are the absence of crossterms between diffusion and localization gradients, a very clean, selfrefocused LASER localization, and greater flexibility of the sequence. Using such a block-based scheme, the diffusion block can be readily designed according to specific experimental questions, independently of the localization block. For example, a Double Diffusion Encoding (DDE) scheme could be implemented using this approach (Shemesh et al., 2014b), giving rise to excellent SNR that enabled measurements of metabolite microscopic anisotropy (Fig. 3); a stimulated-echo scheme was implemented in Ligneul et al. (2017) to reach very high b values and study how metabolite signal attenuation depended on TE and TM without any bias due to cross-terms (Fig. 2D). Shemesh et al. exploited spectrally-selective excitation and refocusing to precisely control the spectral profile, manipulating only metabolites/chemical shifts of interest independently of the localization itself. Spectra acquired using this approach were termed Relaxation Enhanced MRS (RE MRS), as they benefit from the absence of water signal and the potential gain in signal enhancements due to relaxation enhancement arising from potential cross relaxation effects between metabolites and water. Adapting this approach to clinical systems remains to be done and might be difficult with a LASER localization block (because of the SAR and of the relatively long TE that the block design would impose), but an ISIS (Image-Selected In vivo Spectroscopy (Ordidge et al., 1986)) localization block may be used instead to overcome these limitations. It is important to mention that a small diffusion-weighting is generated by the localization block $\left(\sim 0.5 \mathrm{~ms} / \mu \mathrm{m}^{2}\right.$, for example see Ligneul et al. (2017)). However, unlike cross-terms, this constant $\mathrm{b}$ term disappears when taking the log of signal attenuation, e.g. when measuring the ADC. In fact, this is one of the advantages of using such separated approaches. However, if one prefers, it can also be readily taken into account for computation of the exact $\mathrm{b}$-value, or the b-value generated by the diffusion block can be reduced accordingly.

Whatever the sequence used, an important constraint arising from the low concentration of metabolites (typically $\sim 10,000$ times less) than water, is that voxel size and acquisition time generally need to be increased compared to DW-MRI, which is a major hurdle to the applicability of DW-MRS. In the rodent brain for example, using high field scanners and state-of-the-art RF coils, acquiring a single b-value along a 


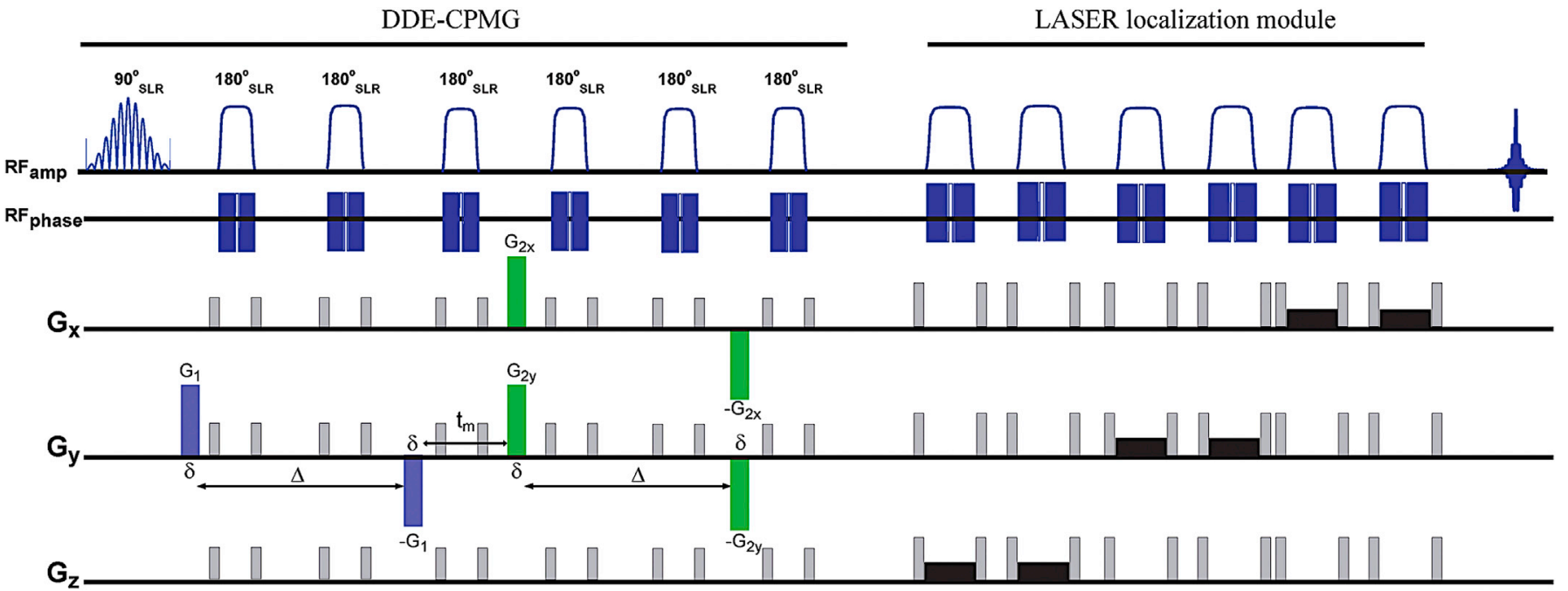

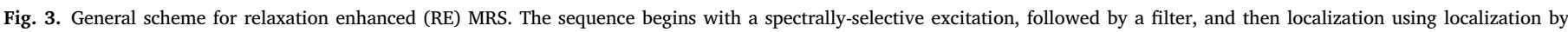

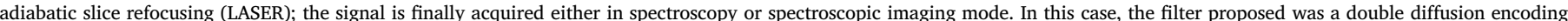

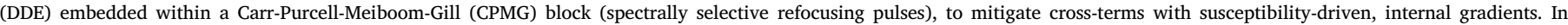

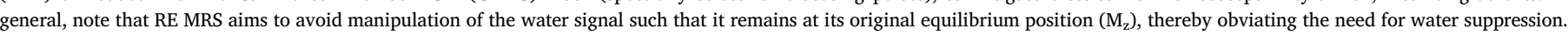

single direction requires a few minutes to get decent SNR value in a voxel of a few $\mu \mathrm{L}$ (i.e. corresponding to a given cerebral structure, such as the hippocampus or the striatum), and acquisition time needs to be increased up to $\sim 1 \mathrm{~h}$ to acquire one spectrum at very long diffusion time (longer than the relaxation time). In Humans, where acquisition time is critical, temporal resolutions of a few minutes are compatible with spatial resolutions of a few cubic centimeters. For example, diffusion tensor MRS was performed on a $3 \mathrm{~T}$ scanner equipped with $20 \mathrm{mT} / \mathrm{m}$ gradients, in $\sim 10 \mathrm{~cm}^{3}$ voxels in the Human brain, using six directions with two bvalues, within $\sim 40$ min using a STEAM sequence (Ellegood et al., 2006) and a PRESS sequence (Ellegood et al., 2011). More recently, a PRESS-based diffusion tensor MRS protocol was performed on a $7 \mathrm{~T}$ scanner equipped with $40 \mathrm{mT} / \mathrm{m}$ gradients in a $3.6 \mathrm{~cm}^{3}$ voxel in Human corpus callosum, using six directions with two b-values in addition to the reference spectrum at $b=0$, within $15 \min$ (Wood et al., 2012).

\section{Post-processing: from raw data to unbiased signal attenuation}

Compared to DW-MRI, additional sources of bias can lead to incorrect quantification of DW-MRS signal attenuation. Fortunately, most of these sources of bias can be accounted for, or at least partially controlled. Key post-processing steps to obtain "unbiased" signal attenuation have already been detailed in a recent review (Ronen and Valette, 2015), but will nonetheless be briefly reviewed here, as unbiased measurement is critical in the perspective of microstructure modeling.

Bulk translational motion in the presence of diffusion gradients results in signal phase variations. This is problematic in MRS, because many scans are generally averaged to get sufficient SNR, and averaging of spectra with different phases will result in overall signal attenuation and/ or distortion. If enough metabolite signal can be detected in a single transient, phase correction performed directly on each transient prior to averaging has been shown to be effective to overcome this artefact (Posse et al., 1993). Alternatively, if metabolite signal is too low, residual water signals may be used, or the full water signal when metabolite cycling is performed (Adalid et al., 2017; Dreher et al., 2001). However, care should be taken when using the water signal as a phase correction reference, as it does not necessarily originate from the same compartment, in particular if the voxel contains CSF. In such cases, phase correction based on water may not fully restore metabolite phase. To circumvent this problem, it has been proposed to use the water signal after adding an inversion-recovery CSF-nulling block (Adalid et al.,
2017). Other kinds of bulk motion, such as rotational motion, result in overall signal attenuation on individual scans, and are therefore less trivial to correct. If sufficient signal is available on single scans, it is possible to select and discard scans exhibiting abnormal signal attenuation e.g., below a certain threshold determined from the highest signal outcome of the series and taking SNR into account. Macromolecule signal at $0.9 \mathrm{ppm}$, which does not overlap with metabolite signal, has been used in a recent study using oscillating gradients (Ligneul and Valette, 2017) as a reference of approximately null diffusivity, at least at low b-values, so that scans with decreased MM signal compared with $b=0$ could be discarded as presumably resulting from bulk motion artefact.

The effect of eddy currents on spectral distortion has been shown to be relatively easy to correct for Ordidge et al. (1986), provided a water reference signal was acquired using the same sequence, i.e. data are acquired with the same volume selection, same sequence timing and the same diffusion-weighting, with water-suppression RF pulses turned off. Assuming only the water resonance contributes to this reference signal (which is generally the case when no water suppression is applied and diffusion-weighting is not too high), the temporal phase distortion induced by eddy current can be directly assessed by measuring the phase $\varphi_{\mathrm{EC}}(\mathrm{t})$ of the free induction decay. Then, eddy currents correction on the water-suppressed free induction decay $\operatorname{FID}_{\text {metab }}(t)$ is achieved by simply removing the eddy current-induced phase, i.e. by computing FID $_{\text {metab, }}$ $\operatorname{ECC}(\mathrm{t})=\mathrm{FID}_{\text {metab }}(\mathrm{t}) / \exp \left(i \varphi_{\mathrm{EC}}(\mathrm{t})\right)$. This approach is compromised in situations where metabolite signal significantly contributes to the reference signal acquired without water suppression, for example at very high $b$ values. In that case, a linear-prediction singular value decomposition (LPSVD) algorithm allows isolating the water signal before calculating $\varphi_{\text {EC }}(t)$ (Vanhamme et al., 1999). This approach may also be useful in poor SNR conditions.

Once MRS spectra have been properly reconstructed, metabolite signal needs to be quantified. This was historically done by measuring peak height, area, or ratios, which can be accurate when the baseline is flat and when the different metabolites do not overlap. However, to measure diffusion properties for as many metabolites as possible, including J-modulated metabolite such as Glu and Ins, short TE sequences are required, resulting in many overlapping multiplets as well as strong macromolecule contribution (see below). In such cases, it is much more reliable to analyze spectra in terms of linear combination of individual metabolite signals (Fig. 4), as performed, e.g., by LCModel (Provencher, 1993). Other analysis software exist, such as AMARES 

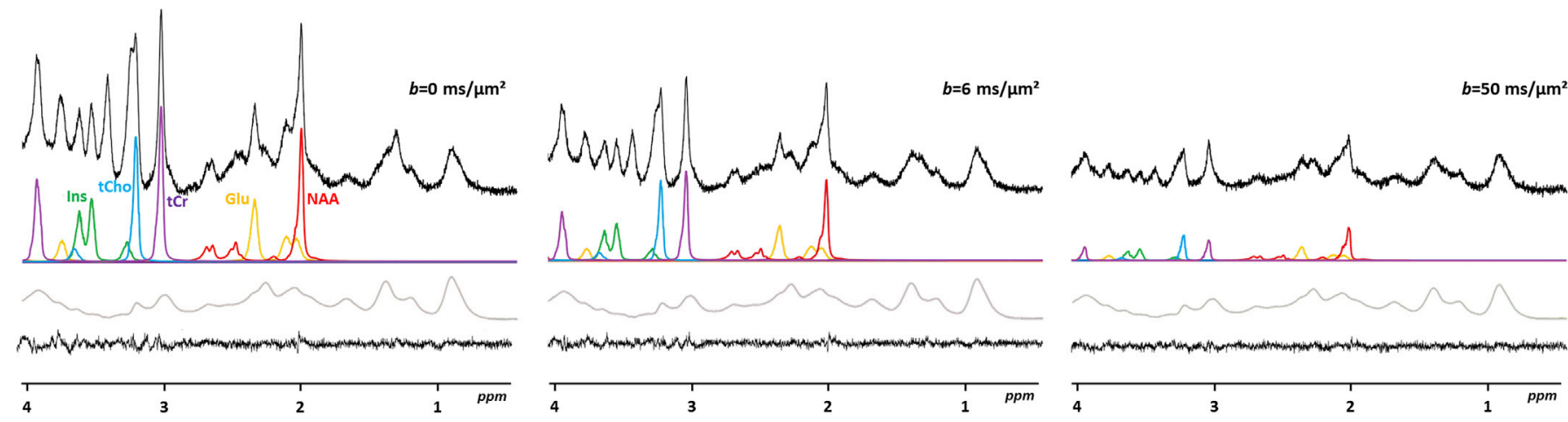

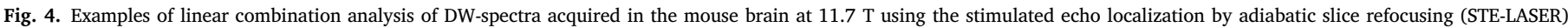

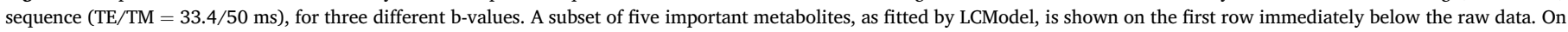

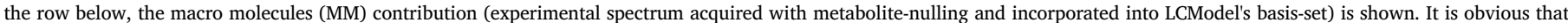

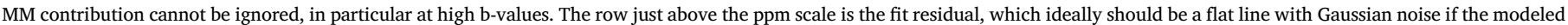
spectrum can perfectly explain the data.

(Mierisova et al., 1998), TARQUIN (Wilson et al., 2011), FID-A (www. github.com/CIC-methods/FID-A), and these also quantify spectra in similar ways. This permits the utilization of the exhaustive spectral information, in particular by simultaneously fitting peaks at different chemical shifts but belonging to the same metabolite. In the end, it is critical to obtain very robust signal quantification, with more stringent quality criteria than for standard MRS. For example, when using LCModel or TARQUIN, quantification precision may be evaluated based on the Cramér-Rao lower bounds (CRLB). CRLB is an estimation of the standard deviation of the measurement. When measuring the ADC with a two-point experiment (e.g. $b=0$ and $b=2 \mathrm{~ms} / \mu \mathrm{m}^{2}$ ), error propagation analysis yields the following expression for the standard deviation on ADC, as expressed in terms of CRLB provided by LCModel:

$\sigma_{A D C} \sim \frac{1}{b} \times \sqrt{C R L B_{b=2}^{2}+C R L B_{b=0}^{2}}$

Assuming $\mathrm{CRLB}_{\mathrm{b}=2} \sim \mathrm{CRLB}_{\mathrm{b}=0}-20 \%$, often considered as a reliability threshold in the MRS literature, we get s.d.(ADC) $\sim 0.14 \mu \mathrm{m}^{2} / \mathrm{ms}$, which is comparable to a typical ADC. In contrast, a more demanding quality threshold, i.e. CRLB $\sim 5 \%$, will result in s.d.(ADC) $\sim 0.035 \mu \mathrm{m}^{2} / \mathrm{ms}$, which is well below typical ADC.

A very important and often overlooked issue related to metabolite quantification is the macromolecule (MM) signal. MM consist of large molecules, e.g. proteins, with relatively short $\mathrm{T}_{2}$ (a few tens of $\mathrm{ms}$ ) resulting in broad resonances overlapping with metabolites (Behar and Ogino, 1993). Not surprisingly, MM diffusion has been reported to be very slow, with ADC in the brain found to be $\sim 0.005-\sim 0.01 \mu \mathrm{m}^{2} / \mathrm{ms}$ and a close to mono-exponential attenuation even at very large b-values (Ligneul et al., 2017; Pfeuffer et al., 2000). MM signal is not negligible compared to metabolites when relatively short TE sequences are used (typically less than $50 \mathrm{~ms}$ ), and it has been shown to significantly affect estimated metabolite diffusion if not properly accounted for Valette et al. (2005), especially at higher b-values where MM have a higher relative signal due to their slow diffusion (Fig. 4). In this context, it is absolutely critical to carefully disentangle metabolite from MM signal. The spectral quantification software LCModel (Provencher, 1993) offers the possibility to include a group of independent broad contributions to model MM signal, but this will generally introduce too much bias and variability, as overlapping MM and metabolite signal can still be confounded. The method of choice is instead to acquire an experimental MM spectrum using metabolite-nulling acquisition (Behar et al., 1994) and to incorporate this spectrum in LCModel basis-set. This will ensure that the actual MM contribution is considered, thus limiting the variability in the estimation of the MM signal at different $b$ values, in particular because the isolated MM peak at $0.9 \mathrm{ppm}$ will result in reliable MM estimation.
Early measurements of metabolite DW-MRS signal decays, ADC and diffusion tensor MRS

Somewhat similarly to the history of water-based DWI, the initial focus in DW-MRS was in characterizing the signal attenuation, and measuring metabolite ADCs at a given set of diffusion time and gradient amplitude combination using Single Diffusion Encoding (SDE) approaches (Shemesh et al., 2016). In perfused cells, van Zijl et al. already identified non-exponential decays against b-value (Vanzijl et al., 1991), while Moonen et al. measured metabolite ADCs in muscle tissue (Moonen et al., 1990). Several metabolite ADCs were subsequently measured in the healthy human brain (Posse et al., 1993; Ellegood et al., 2011, 2006, 2005) as well as in the in-vivo rat brain (Merboldt et al., 1993; Nicolay et al., 1995), reporting ADC values of the three main metabolites: NAA, $\mathrm{tCr}$ and tCho, consistent between human and rat brain and around $0.15-0.20 \mu \mathrm{m}^{2} / \mathrm{ms}$. For a summary table of ADC values in various brain regions and animal species, we refer to the comprehensive Table 1 in Cao and Wu (2017).

Wick et al. (1995) monitored changes in metabolite ADCs upon ischemia in the rat, and suggested that specific neuronal and astrocytic swellings could be identified from the NAA and Ins signals, respectively. van der Toorn et al. (1996) measured a significant decrease in NAA and tCr ADCs of $29 \%$ and $19 \%$, respectively, compared to the contralateral region, after $3 \mathrm{~h}$ of ischemia in rat brain. Pfeuffer et al. (2000) were able to use DW-MRS derived ADCs of glucose, lactate and compare them to purely intracellular metabolites to demonstrate the equal partitioning of glucose and lactate across the extra/intra-cellular compartments. Others studied the ADCs in stroke (Dreher et al., 2001; Abe et al., 2000; Nicoli et al., 2003) as well as in tumors (Harada et al., 2002). These early studies all showed that there is value in measuring metabolite diffusion, yet, most of them did not address tissue microstructure directly, but rather indirectly interpreted ADC variations in terms of possible alterations of microstructure.

In analogy to DTI, it is possible to assess the macroscopic anisotropy of metabolite diffusion in the brain tissue by using a minimum of six diffusion gradient directions. This technique is called diffusion tensor spectroscopy (DT-MRS, also known as DTS), and permits the measurement of the elements of the macroscopic diffusion tensor of typical MRSvisible metabolites, such as NAA, tCr and tCho. DT-MRS has been used to assess the anisotropy of metabolite diffusion in human (Ellegood et al., 2006; Upadhyay et al., 2007, 2008), animal model of frog peripheral nerve (Ellegood et al., 2007) and bovine optic nerve (Assaf and Cohen, 1999).

In human brain, elevated fractional anisotropy (FA) values of 0.48-0.72 for NAA (Ellegood et al., 2006; Upadhyay et al., 2007, 2008), 0.56-0.73 for tCr (Ellegood et al., 2006) and 0.59-0.74 for tCho 
Table 1

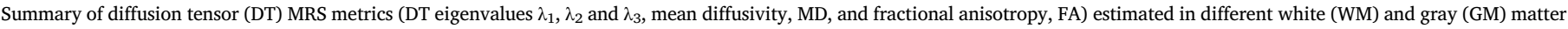
regions in healthy human brain together with the corresponding references.

\begin{tabular}{|c|c|c|c|c|c|c|c|}
\hline \multirow[t]{2}{*}{ System } & \multirow[t]{2}{*}{ Sequence parameters } & \multirow[t]{2}{*}{ Metabolite } & \multicolumn{5}{|l|}{ DT-MRS } \\
\hline & & & $\begin{array}{l}\lambda_{1}\left(\mu m^{2} /\right. \\
m s)\end{array}$ & $\begin{array}{l}\lambda_{2}\left(\mu m^{2} /\right. \\
m s)\end{array}$ & $\begin{array}{l}\lambda_{3}\left(\mu m^{2} /\right. \\
m s)\end{array}$ & $\begin{array}{l}M D\left(\mu m^{2} /\right. \\
m s)\end{array}$ & FA \\
\hline Human Brain $W M+G M$ & $\mathrm{~B}_{0}=3 \mathrm{~T} ; \mathrm{TE}=90 \mathrm{~ms}$ & NAA & $/ /$ & // & // & $0.17 \pm 0.04$ & $0.57 \pm 0.08$ \\
\hline \multirow[t]{2}{*}{ (Fotso et al., MRM, 2017) } & $\mathrm{b}_{\max }=1.7 \mathrm{~ms} / \mu \mathrm{m}^{2}$ & $\mathrm{tCr}$ & $/ /$ & // & // & $0.20 \pm 0.04$ & $0.63 \pm 0.04$ \\
\hline & $\mathrm{td}=45 \mathrm{~ms}$ & tCho & $/ /$ & $/ /$ & $/ /$ & $0.18 \pm 0.04$ & $0.57 \pm 0.10$ \\
\hline Human Brain Occipital GM & $\mathrm{B}_{0}=3 \mathrm{~T} ; \mathrm{TE}=160 \mathrm{~ms}$ & NAA & $0.35 \pm 0.07$ & $0.22 \pm 0.05$ & $0.10 \pm 0.06$ & $0.22 \pm 0.05$ & $0.53 \pm 0.14$ \\
\hline \multirow[t]{2}{*}{ (Ellegood et al., NMR Biomed, 2011) } & $\mathrm{b}_{\max }=1.8 \mathrm{~ms} / \mu \mathrm{m}^{2}$ & $\mathrm{tCr}$ & $0.38 \pm 0.07$ & $0.22 \pm 0.08$ & $0.07 \pm 0.02$ & $0.22 \pm 0.05$ & $0.60 \pm 0.10$ \\
\hline & $\mathrm{td}=75 \mathrm{~ms}$ & tCho & $0.35 \pm 0.08$ & $0.22 \pm 0.07$ & $0.09 \pm 0.07$ & $0.22 \pm 0.05$ & $0.54 \pm 0.13$ \\
\hline Human Brain Subcortical WM & $\mathrm{B}_{0}=3 \mathrm{~T} ; \mathrm{TE}=160 \mathrm{~ms}$ & NAA & $0.38 \pm 0.06$ & $0.24 \pm 0.03$ & $0.15 \pm 0.04$ & $0.26 \pm 0.01$ & $0.43 \pm 0.13$ \\
\hline \multirow[t]{2}{*}{ (Ellegood et al., NMR Biomed, 2011) } & $\mathrm{b}_{\max }=1.8 \mathrm{~ms} / \mu \mathrm{m}^{2}$ & $\mathrm{tCr}$ & $0.39 \pm 0.05$ & $0.22 \pm 0.02$ & $0.12 \pm 0.04$ & $0.24 \pm 0.02$ & $0.51 \pm 0.11$ \\
\hline & $\mathrm{td}=75 \mathrm{~ms}$ & tCho & $0.36 \pm 0.07$ & $0.20 \pm 0.03$ & $0.09 \pm 0.04$ & $0.22 \pm 0.02$ & $0.54 \pm 0.15$ \\
\hline $\begin{array}{l}\text { Human Brain Corpus Callosum Body (Ellegood et al., NMR } \\
\text { Biomed, 2011) }\end{array}$ & $\begin{array}{l}\mathrm{B}_{0}=3 \mathrm{~T} ; \mathrm{TE}=160 \mathrm{~ms} \\
\mathrm{~b}_{\max }=1.8 \mathrm{~ms} / \mu \mathrm{m}^{2} ; \\
\mathrm{td}=75 \mathrm{~ms}\end{array}$ & NAA & $0.38 \pm 0.01$ & $0.24 \pm 0.02$ & $0.09 \pm 0.02$ & $0.23 \pm 0.01$ & $0.55 \pm 0.06$ \\
\hline $\begin{array}{l}\text { Human Brain Corticospinal Tract } \\
\text { (Ellegood et al., NMR Biomed, 2011) }\end{array}$ & $\begin{array}{l}\mathrm{B}_{0}=3 \mathrm{~T} ; \mathrm{TE}=160 \mathrm{~ms} \\
\mathrm{~b}_{\max }=1.8 \mathrm{~ms} / \mu \mathrm{m}^{2} ; \\
\mathrm{td}=75 \mathrm{~ms}\end{array}$ & NAA & $0.51 \pm 0.13$ & $0.32 \pm 0.05$ & $0.08 \pm 0.01$ & $0.31 \pm 0.06$ & $0.52 \pm 0.03$ \\
\hline $\begin{array}{l}\text { Human Brain Left Arcuate Fasciculus (Upadhyay et al., } \\
\text { NeuroImage, 2008) }\end{array}$ & $\begin{array}{l}\mathrm{B}_{0}=3 \mathrm{~T} ; \mathrm{TE}=135 \mathrm{~ms} \\
\mathrm{~b}_{\max }=1.7 \mathrm{~ms} / \mu \mathrm{m}^{2} ; \\
\mathrm{td}=50 \mathrm{~ms}\end{array}$ & NAA & $0.27 \pm 0.01$ & $\begin{array}{l}\left(\lambda_{2}+\lambda_{3}\right) / 2= \\
\mu \mathrm{m}^{2} / \mathrm{ms}\end{array}$ & $.12 \pm 0.01$ & $0.20 \pm 0.01$ & $0.53 \pm 0.06$ \\
\hline $\begin{array}{l}\text { Human Brain Right Arcuate Fasciculus (Upadhyay et al., } \\
\text { NeuroImage, 2008) }\end{array}$ & $\begin{array}{l}\mathrm{B}_{0}=3 \mathrm{~T} ; \mathrm{TE}=135 \mathrm{~ms} \\
\mathrm{~b}_{\max }=1.7 \mathrm{~ms} / \mu \mathrm{m}^{2} ; \\
\mathrm{td}=50 \mathrm{~ms}\end{array}$ & NAA & $0.25 \pm 0.01$ & $\begin{array}{l}\left(\lambda_{2}+\lambda_{3}\right) / 2= \\
\mu \mathrm{m}^{2} / \mathrm{ms}\end{array}$ & $.14 \pm 0.01$ & $0.20 \pm 0.01$ & $0.43 \pm 0.02$ \\
\hline $\begin{array}{l}\text { Human Brain Corpus Callosum Body } \\
\text { (Ronen et al., Brain Struct. Func. 2014) }\end{array}$ & $\begin{array}{l}\mathrm{B}_{0}=7 \mathrm{~T} ; \mathrm{TE}=120 \mathrm{~ms} \\
\mathrm{~b}_{\max }=3.6 \mathrm{~ms} / \mu \mathrm{m}^{2} ; \\
\mathrm{td}=50 \mathrm{~ms}\end{array}$ & NAA & $0.34 \pm 0.03$ & $0.24 \pm 0.03$ & $0.08 \pm 0.01$ & $0.22 \pm 0.02$ & $0.54 \pm 0.04$ \\
\hline Human Brain Occipital GM & $\mathrm{B}_{0}=3 \mathrm{~T} ; \mathrm{TE}=160 \mathrm{~ms}$ & NAA & $0.23 \pm 0.03$ & $0.18 \pm 0.03$ & $0.14 \pm 0.03$ & $0.18 \pm 0.02$ & $0.25 \pm 0.10$ \\
\hline \multirow[t]{2}{*}{ (Ellegood et al., NMR Biomed, 2011) } & $\mathrm{b}_{\max }=5 \mathrm{~ms} / \mu \mathrm{m}^{2}$ & $\mathrm{tCr}$ & $0.24 \pm 0.03$ & $0.19 \pm 0.02$ & $0.13 \pm 0.03$ & $0.19 \pm 0.02$ & $0.30 \pm 0.07$ \\
\hline & $\mathrm{td}=75 \mathrm{~ms}$ & tCho & $0.21 \pm 0.03$ & $0.17 \pm 0.02$ & $0.12 \pm 0.03$ & $0.17 \pm 0.03$ & $0.28 \pm 0.06$ \\
\hline Human Brain Subcortical WM & $\mathrm{B}_{0}=3 \mathrm{~T} ; \mathrm{TE}=160 \mathrm{~ms}$ & NAA & $0.33 \pm 0.08$ & $0.19 \pm 0.03$ & $0.12 \pm 0.02$ & $0.21 \pm 0.02$ & $0.47 \pm 0.13$ \\
\hline \multirow[t]{2}{*}{ (Ellegood et al., NMR Biomed, 2011) } & $\mathrm{b}_{\max }=5 \mathrm{~ms} / \mu \mathrm{m}^{2}$ & $\mathrm{tCr}$ & $0.32 \pm 0.07$ & $0.19 \pm 0.02$ & $0.09 \pm 0.02$ & $0.20 \pm 0.02$ & $0.51 \pm 0.13$ \\
\hline & $\mathrm{td}=75 \mathrm{~ms}$ & tCho & $0.26 \pm 0.06$ & $0.15 \pm 0.05$ & $0.08 \pm 0.01$ & $0.16 \pm 0.02$ & $0.51 \pm 0.14$ \\
\hline $\begin{array}{l}\text { Human Brain Corpus Callosum Body (Ellegood et al., NMR } \\
\text { Biomed, 2011) }\end{array}$ & $\begin{array}{l}\mathrm{B}_{0}=3 \mathrm{~T} ; \mathrm{TE}=160 \mathrm{~ms} \\
\mathrm{~b}_{\max }=5 \mathrm{~ms} / \mu \mathrm{m}^{2} ; \\
\mathrm{td}=75 \mathrm{~ms}\end{array}$ & NAA & $0.34 \pm 0.01$ & $0.19 \pm 0.00$ & $0.08 \pm 0.02$ & $0.21 \pm 0.01$ & $0.56 \pm 0.03$ \\
\hline $\begin{array}{l}\text { Human Brain Corticospinal Tract } \\
\text { (Ellegood et al., NMR Biomed, 2011) }\end{array}$ & $\begin{array}{l}\mathrm{B}_{0}=3 \mathrm{~T} ; \mathrm{TE}=160 \mathrm{~ms} \\
\mathrm{~b}_{\max }=5 \mathrm{~ms} / \mu \mathrm{m}^{2} ; \\
\mathrm{td}=75 \mathrm{~ms}\end{array}$ & NAA & $0.34 \pm 0.04$ & $0.22 \pm 0.02$ & $0.15 \pm 0.11$ & $0.24 \pm 0.06$ & $0.41 \pm 0.19$ \\
\hline
\end{tabular}

(Ellegood et al., 2006) were observed in major WM regions, such as the corpus callosum, corticospinal tract and arcuate fasciculus. These tensor measurements agreed with specific measurements of anisotropic diffusion of NAA in the human corpus callosum (Kroenke et al., 2004), as well as in animal models of frog peripheral nerve (Ellegood et al., 2007) and bovine optic nerve (Assaf and Cohen, 1999). Interestingly, a first examination of cortical gray matter (GM) yielded an unexpected high degree of anisotropy (FA $=0.53-0.79)$ for all three metabolites, similar to the WM FA values (Ellegood et al., 2006). In contrast, typical FA values of water in cortical GM are approximately 0.2 (Bhagat and Beaulieu, 2004) or less. A later study by Ellegood et al. (2011) showed that the high FA measured in GM was an artefact caused by the variability of the directional ADC measurements (Chang et al., 2007). The greater directional ADC variability in cortical GM was the result of a poor two-point slope estimation of the signal intensity versus b value when using a less than ideal maximum $b$ value of $3-5 \mathrm{~ms} / \mu \mathrm{m}^{2}$. Using a maximum $b$ value of $\sim 5 \mathrm{~ms} / \mu \mathrm{m}^{2}$, instead of the much lower $1.8 \mathrm{~ms} / \mu \mathrm{m}^{2}$ used in Ellegood et al. (2006), Ellegood et al. estimated lower FA values of $0.25,0.30$ and 0.28 for NAA, tCr and tCho, respectively, in human occipital GM, while FA values of $0.47,0.51$ and 0.51 for NAA, $\mathrm{tCr}$ and tCho, respectively, in human subcortical WM were consistent with the estimates at maximal $b$ of $1.8 \mathrm{~ms} / \mu^{2}{ }^{2}$ (Ellegood et al., 2011). A summary of DT-MRS metrics (DT eigenvalues $\lambda_{1}, \lambda_{2}$ and $\lambda_{3}$, mean diffusivity, MD, and FA) estimated in different WM and GM regions in healthy human brain are reported in Table 1. The DT-MRS metrics estimated by different research groups with different scanners and different magnetic fields, but similar echo time and diffusion time, show a good consistence and reproducibility (Table 1).
What does DW-MRS data tell about brain cells microstructure?

Metabolite ADC and DT derived metrics, while very useful in some cases, are only indirect reporters of microstructure as they reflect the overall macroscopic reduction in diffusion imposed by geometry in the Gaussian diffusion limit. Earlier strategies for quantifying microstructure from metabolite DW-MRS have involved q-space MRS (Assaf and Cohen, 1998a, 1998b), which clearly showed non-monoexponential diffusion for NAA, and even quantified some of its time-dependent properties by observing the average propagator at different diffusion times. Others proposed a first attempt to model NAA diffusion taking into account the cellular structure (Yablonskiy and Sukstanskii, 2010; Kroenke et al., 2004), while more recently, the ADC time dependence at (ultra)-short td was linked to local cellular geometry such as fiber diameter (Marchadour et al., 2012; Ligneul and Valette, 2017), while the ADC at (ultra-)long $t_{d}$ was linked to the long-range cell morphology (Palombo et al., 2016).

In this section, the most recent frameworks proposed to relate brain metabolites diffusion in the intra-cellular space to microstructure are reviewed, together with some examples of recent successful applications to experimental data, which support their validity. We focus our interest on geometrical models which link the measured diffusion-sensitized echo signal attenuation and/or derived diffusion metrics, such as ADC, to cellular microstructural determinants, such as fibers diameter and length, number of embranchments and others.

\section{Molecular diffusion and DW-MR signal}

Conventional spin-echo and stimulated-echo MRI and MRS can be 
sensitized to diffusion by symmetrically applying magnetic field gradient pulses that attenuate the echo signal S. In the case of non-restricted or Gaussian diffusion, the effect of molecular diffusion leads to an exponential attenuation of $\mathrm{S}$ with the b-value. However, in the general case of diffusion in biological tissue, the presence of restrictive or hindering boundaries (membranes, cytoskeleton, macromolecules, organelles etc ...) drastically influences the motion of probe molecules (water or metabolites) and the consequent signal attenuation and the derived ADC. In particular, the echo signal attenuation $S$ is no longer a simple exponential decay and the measured ADC is in this case depends on $t_{d}$ (Grebenkov, 2007; Latour et al., 1994; Sen, 2004; Novikov et al., 2014).

In the case of water diffusion, the description and interpretation of the measured signal and derived metrics such as the ADC in terms of the underlying tissue microstructure is generally very difficult, due to the non-specificity of the water signal and the complexity of the tissue as a whole. Non-negligible water volume fraction in the extracellular space, whose geometric properties can be very difficult to assess, as well as cell membrane permeability have to be taken into account. Phenomena related to the extracellular space, such as intra-/extra-cellular water exchange, potential flow of the cerebrospinal fluid (CSF), extracellular volume fraction, diffusivity and tortuosity in the extracellular space, cannot be neglected for water signals, but can be neglected when interpreting and modeling intracellular metabolite diffusion. For some metabolites, diffusion properties are expected to depend mostly on intracellular parameters such as cytosol viscosity, molecular crowding and binding, as well as the size and shape of the cellular compartment, and metabolite diffusion modeling is thus much simpler, allowing for a more direct and precise estimate of the specific cellular compartment features.

\section{Modeling metabolite intra-cellular diffusion}

It is important to distinguish the case of restricted diffusion from hindered diffusion, because the echo signal attenuation and the ADC diffusion time dependence for these two scenarios are different.

\section{Hindering effects: tortuosity and obstruction}

Numerous immobile obstacles (i.e. considering the typical time window of DW-MRS experiments) exist within the intracellular space, such as the cytoskeleton and various organelles, potentially making the cytosol a tortuous space. The tortuosity $\tau$ refers to the effect of hindrances imposed by various immobile obstacles in the medium on the path that diffusing molecules can take. Such hindrances affect the minimal pathway between two points so that the pathway becomes tortuous, rather than a straight line. In this case, the shortest pathway between two points is increased, on average, by a factor $\tau(\geq 1)$, compared to a straight line connecting these points. At very short $t_{d}$, during which the mean square displacement (MSD) is very small compared to the square of the typical distance between obstacles, tortuosity will not affect molecular displacement, and the diffusion process will appear free. At longer $t_{d}$, in d dimensions, one has (Price, 2009):

$M S D \sim 2 d \frac{D_{\text {free }}}{\tau^{2}} t_{d}$

Equivalently, the ADC measured at long $t_{d}$ converges to: $\lim _{t_{d} \rightarrow \infty} A D C=A D C_{\infty} \sim \frac{D_{\text {free }}}{\tau^{2}}$

The way in which the ADC approaches its tortuosity limit and the value of the tortuosity itself in Eq. (3) may be affected by secondary structures of cell morphology such as dendritic spines, astrocytic leaflets or axonal beads (Ligneul et al., 2017). These secondary structures can be seen as randomly distributed hindering sources to metabolites diffusion along cell fibers. In this case, a well-behaved and specific ADC power law time dependence is expected for mono-dimensional short-range disordered (hindering) structures: ADC $\sim \mathrm{ADC} \infty+\mathrm{Ct}_{\mathrm{d}}^{-0.5}$ (Novikov et al., 2014). Recent numerical simulations and experimental results (Ligneul et al., 2017) suggest that structures such as these can also affect metabolite diffusion and the measured echo signal attenuation at high $\mathrm{q} / \mathrm{b}$ values. Consequently, they have a non-negligible effect on the derived diffusion metrics, such as ADC power law time dependence, and on the estimated cell geometrical parameters such as fiber radius (Ligneul et al., 2017).

A related but nevertheless distinct phenomenon compared with tortuosity that can also lead to a decrease in the measured ADC is that of obstruction. In cell cytoplasm, macromolecules cannot be neglected compared to the solvent concentration, thus smaller molecules (e.g., metabolites) have to skirt around the larger and generally irregularly shaped obstructing molecules, increasing their diffusion path length. Obstruction is a complicated many-body problem which is in essence hindrance by a time-dependent geometry - the obstructing molecules are also moving - in which the interactions between the particles need to be considered. Obstruction results in the measured ADC being reduced by a factor that depends on the concentration of the particles, as well as perhaps by other factors, such as electrolyte friction and solvation (Price, 2009; Allison et al., 2001).

The effects leading to obstruction generally operate on very short $t_{d}$ and length scales and are consequently typically well averaged on experimentally available timescales. Thus, from the perspective of diffusion, obstruction and viscosity are similar in their effects and closely related, and this helps to explain why the measured viscosity, which includes obstruction by macromolecules, can depend on the size of the probe molecule (Price et al., 1989).

\section{Restriction effects}

The confinement of metabolites within a given compartment, such as organelles or even the entire intracellular space, will also impose an upper limit to the displacement variance. While this restriction effect is negligible at very short $t_{d}$, as the displacement variance is very small compared to the square of the typical distance between diffusion barriers, at long $t_{d}$, restriction will strongly influence the diffusion process. For example, in a situation of perfect restriction, i.e., without a possible escape for diffusing molecules, the ADC will converge to 0 with increasing $t_{d}$, thus, the convergence of the ADC to zero with increasing $t_{d}$ is a specific signature of restricted diffusion. Similarly, the echo signal attenuation as a function of $b$ deviates from the simple exponential decay. For example, for diffusion within a reflective cylinder of radius $a$ and when the diffusion sensitizing gradient is applied along the direction orthogonal to the restricting frontiers, in the short gradient pulses (SGP) approximation (Soderman and Jonsson, 1995) the signal attenuation is given by:

$\frac{S_{c y l i n d e r}\left(q, t_{d}\right)}{S\left(q=0, t_{d}\right)}=\frac{\left[2 J_{1} 2 \pi q a\right]^{2}}{(2 \pi q a)^{2}}+8(2 \pi q a)^{2} \sum_{n=1}^{\infty}\left\{\frac{1}{1+\delta_{n 0}}\left[J_{n}^{\prime} 2 \pi q a\right]^{2} \sum_{m=1}^{\infty} \frac{\alpha_{n m}^{2}}{\left(\alpha_{n m}^{2}-n^{2}\right)\left[\alpha_{n m}^{2}-(2 \pi q a)^{2}\right]^{2}} e^{-\frac{D_{\text {frec }} a_{n n^{2} d}^{2}}{a^{2}}}\right\}$ 
where $J_{n}$ is the Bessel function of integer order $n ; \alpha_{n m}$ is the m-th positive root of the Bessel equation $J_{n}^{\prime}=0 ; \delta_{n 0}$ is the Kronecker delta symbol. Note that for other simple restricting geometries like parallel infinite reflective planes or reflective sphere, similar exact analytical solutions have been derived in SGP approximation (Balinov et al., 1993).

As can be inferred from the attenuation behaviour in Eq. (4), as $t_{d}$ is such that the diffusing spins interact with the enclosing geometry (i.e., $t_{d} \sim a^{2} / D_{\text {free }}$ where $a$ is the size of the confining geometry) the attenuation profile differs significantly from that of the free diffusion model. When the interactions with the boundary become significant $\left(t_{d} \gtrsim a^{2}\right.$ / $D_{\text {free}}$ ) an interesting effect is noted if the attenuation is plotted as a function of q: diffusive diffraction-like effects arise and structural information about the enclosing geometry can be obtained from the characteristics of the diffraction pattern. For example, the diffractive minima occur at $\mathrm{q}=\mathrm{n} /(2 \mathrm{a})(\mathrm{n}=1,2,3, \ldots)$. In the early 1990s, Callaghan and Coy proposed for the first time the analogy between NMR measurements in a field gradient and diffraction, formulating its link to the underpinning confining geometry (Callaghan et al., 1991; Callaghan, 1995). Many subsequent DW-MRI and DW-MRS studies of water and metabolite diffusion in biological tissues showed the potential of this approach to characterize tissue microstructure (Assaf and Cohen, 1999; Avram et al., 2004; Bar-Shir et al., 2008). For a comprehensive review on this topic, we refer the reader to Cohen and Assaf. (2002).

For the case of cylindrical restrictions, it is not always easy or even possible to apply the diffusion sensitizing gradient exactly along the direction orthogonal to the restricting frontiers. In this case, an expression for the echo signal attenuation in the generic case where the diffusion gradient is applied along a direction separated by an angle $\theta$ relative to the cylinder axis is provided by (Linse and Soderman, 1995):

$\frac{S_{\text {cylinder }}^{\theta}\left(q, t_{d}\right)}{S\left(q=0, t_{d}\right)}=\frac{S_{\text {cylinder }}\left(q_{\perp}, t_{d}\right)}{S\left(q=0, t_{d}\right)} e^{-D_{\text {free }} q_{\|}^{2} t_{d}}$

where $q_{\perp}=q \sin (\theta) ; q_{\|}=q \cos (\theta)$.

Note that analytical equations also exist to calculate the dispersive diffusivity $\mathrm{D}(\omega)$ (i.e. the Fourier Transform of the velocity autocorrelation function) in cylinders and spheres when using oscillating gradients in the low b-value regime (Stepisnik, 1993). However, one of the great advantage of using oscillating gradients is that, at sufficiently high frequencies (i.e. in the Mitra regime, (Mitra et al., 1993)), a model-free linear fit of $\mathrm{D}(\omega)$ as a function of $\omega$ can be done to estimate $\mathrm{D}_{\text {free }}$ and the surface-to-volume ratio S/V (Novikov and Kiselev, 2011). Such a model-free approach might be preferred over geometrical models, provided sufficiently high $\omega$ can be reached.

\section{Models for cellular compartments}

Generally speaking, the diffusion of brain metabolites in the intracellular space can be modeled as molecular diffusion in the cytosol with restriction mostly due to the cell morphology and internal structure. While the cytosol viscosity, including macromolecular crowding and other effects, can ideally be investigated by performing (ultra-)short $t_{d}$ experiments where the ADC time dependence at (ultra-)short $t_{d}$ can be studied without the necessity of any specific modeling (Ligneul and Valette, 2017), the estimation of cell morphology is more complex, and requires more sophisticated modeling.

Recently obtained experimental results, assist the complex modeling of cellular structure. These results suggest that in order to define a proper model for brain cellular compartment, different diffusion regimes and different corresponding models have to be considered, according to the different $t_{d}$ investigated.

\section{Cytosol viscosity and macromolecular obstruction}

Measurements performed in conditions where tortuosity and restriction effects are assumed to become small or negligible, i.e., at (ultra-) short $t_{d}$ (lower than $10 \mathrm{~ms}$ ) using oscillating gradients (Marchadour et al.,
2012; Ligneul and Valette, 2017) yielded values of metabolite $\mathrm{D}_{\text {free }}$ in the range of $\sim 50 \%$ to $\sim 80 \%$ of the free diffusivity for those metabolites in aqueous solution. This suggests a cytosolic viscosity (including molecular crowding) that is, at most, twice the value of water.

Metabolite diffusion primarily occurs in long fibers: a first argument based on ADC time-dependency

The observed strong decrease in metabolite ADC as $t_{d}$ is increased from $\sim 1 \mathrm{~ms}$ to $\sim 10 \mathrm{~ms}$ reported in Marchadour et al. (2012), Ligneul and Valette (2017) suggests that metabolite diffusion in brain cells is hindered by obstacles that are typically separated by distances of $\leq 2 \mu \mathrm{m}$. A priori, these obstacles could be either organelles or structures of the cytoskeleton, or simply the membranes of fibers extending from the cell bodies of neurons and glial cells, i.e. axons, dendrites and astrocytic processes.

On the other hand, metabolite ADCs (measured within a spectroscopy voxel containing a mix of white and gray matter) have been shown to be remarkably stable in the mouse and macaque brain in the range of (ultra)long $t_{d}$ values between 100 ms and $2 \mathrm{~s}$ (Najac et al., 2014; Palombo et al., 2016), despite a slight trend to decrease with increasing td. This suggests that metabolites are for the most part not confined inside small subcellular structures, such as organelles or cell bodies, but diffuse along the long fibers characteristic of neurons and astrocytes. Had the metabolites been confined to subcellular structures or to a geometrically closed structure such as a cell body, their $\operatorname{ADC}\left(t_{d}\right)$ would have rapidly approached zero. Metabolite ADC stability has also been confirmed separately in human gray and white matter for $t_{d}$ between 100 and 720 ms (Najac et al., 2016).

Thus, brain intracellular metabolites diffusion can be thought primarily as molecular diffusion in cellular processes, described in first approximation as a collection of long cylinders with radius $a \leq 2 \mu \mathrm{m}$, and intracellular metabolites diffusivity $\mathrm{D}_{\text {free }}$ lower than the diffusivity of free metabolites in aqueous solution. The idea of metabolite diffusion being characteristic of diffusion in fibers is consistent with the known cellular morphology of neurons and astrocytes, where long cellular processes (of more than tens, and sometimes up to hundreds of $\mu \mathrm{m}$ ) represent most of the cellular volume fraction, about $80 \%$ or more (Chvatal et al., 2007; Jespersen et al., 2007). Although long axonal fibers may be reasonably considered of infinite length, this assumption may not be valid at all experimentally accessible $t_{d}$ values for dendrites and astrocytic processes which exhibit a more complex branching structure, as will be discussed later in this review.

Metabolite diffusion primarily occurs in long fibers: a second argument based on $D D E$

In contrast to SDE, multiple diffusion encoding approaches - and in particular, Mitra's DDE approach - can provide insights into microscopic anisotropy - a parameter that disentangles orientation distribution from the "local" anisotropy in a heterogeneous system. For more thorough reviews the reader is referred to Finsterbusch (2010), Shemesh et al. (2012), however the main notions will be briefly mentioned here. As its name suggests, the DDE sequence (Fig. 3), first suggested by Cory et al. (1990), contains two diffusion sensitizing epochs separated by a mixing time. Mitra was the first to suggest the angular DDE experiment, a variant with potential to disentangle microscopic from macroscopic anisotropy and orientation dispersion from the shape of the curve spanned by varying the relative angle between DDE's gradient pairs (Mitra, 1995). Mitra thus suggested an ingenious way to characterize the size and shape of completely disordered systems, which would otherwise appear spherical in SDE experiments: spheres would incur no modulation at long mixing times, whereas randomly oriented but locally anisotropic compartments would give rise to a modulation from which the microscopic anisotropy, a measure of the local pore eccentricity, can be extracted. That is, DDE signals, such as those shown in Fig. 5 from a DDE experiment, can have modulations whose amplitude reflects the microscopic anisotropy - in principle, the deeper these oscillations are (as quantified, 
A

${ }^{1} \mathrm{H}$ MRS at $21.1 \mathrm{~T}$

6 seconds acquistion

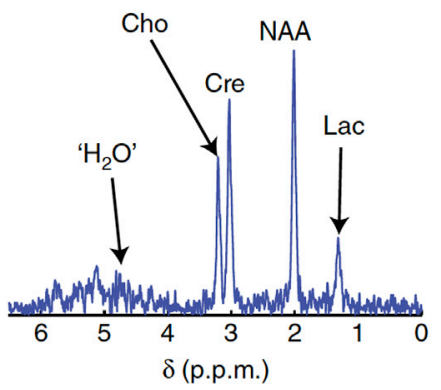

B

C
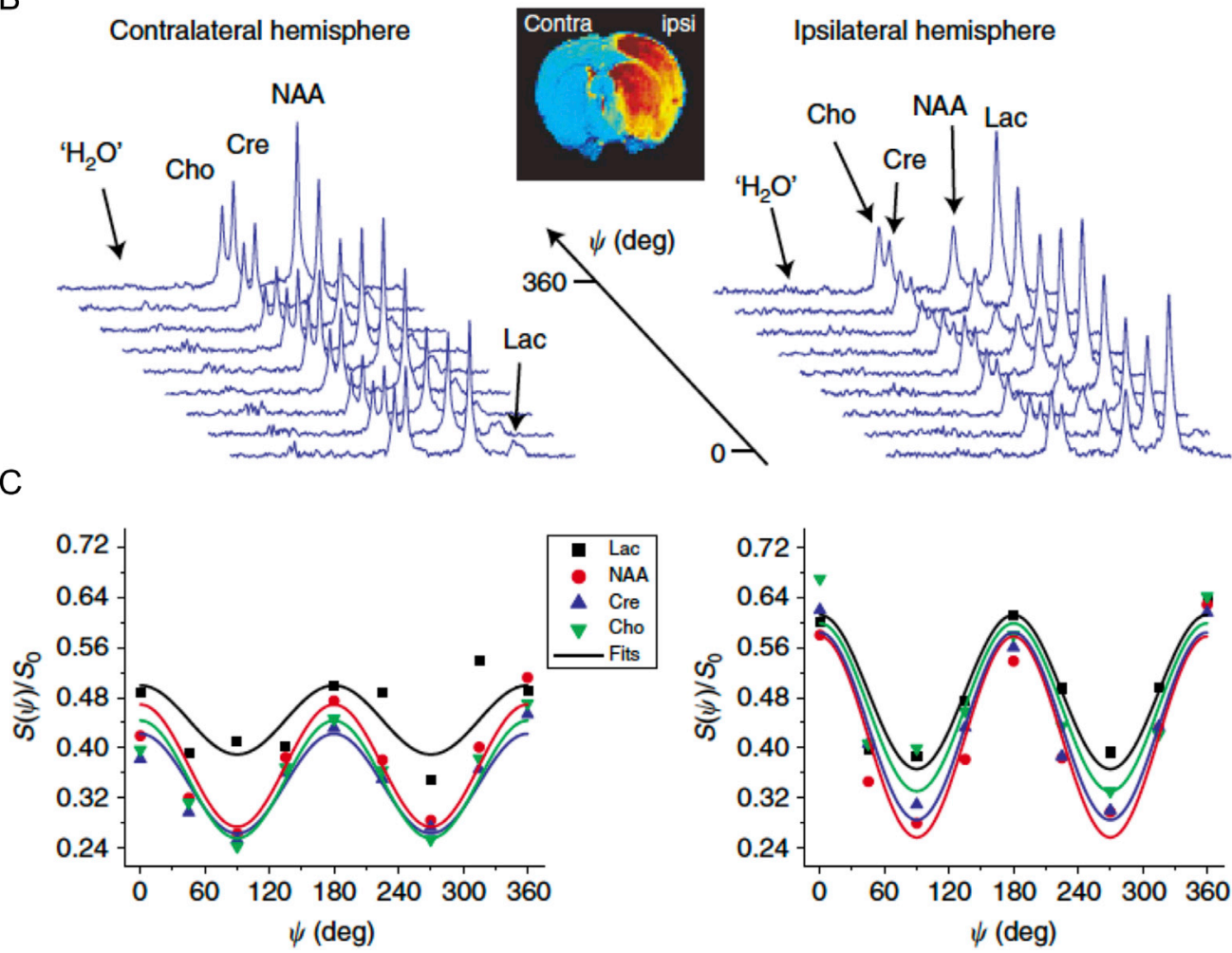

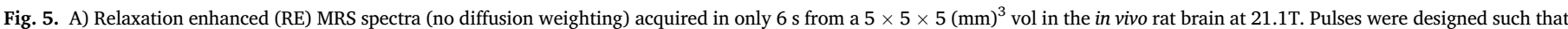

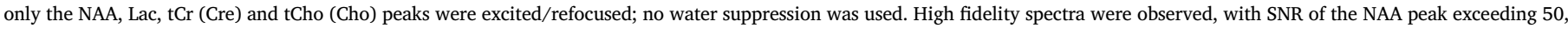

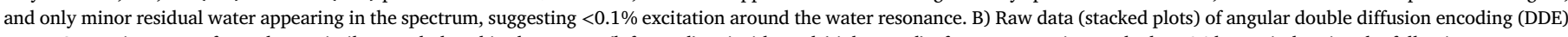

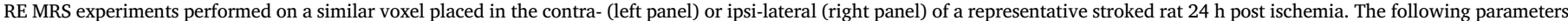

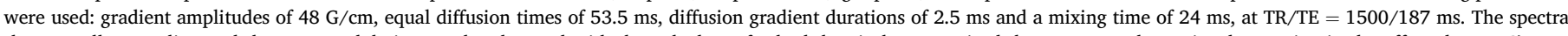

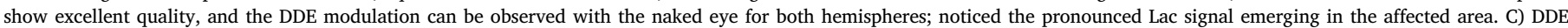

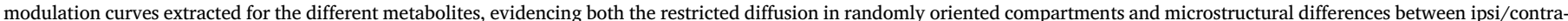
lateral hemispheres after ischemia. For details, see Shemesh et al. (2014b).

e.g., from the signal at 0 and $90^{\circ}$ ), the larger the microscopic anisotropy in the system. It is important to notice that when anisotropic pores are randomly oriented, the SDE experiment provides isotropic decays from which the microscopic anisotropy or orientation dispersion are nearly impossible to recover. This idea was later theoretically refined (Ozarslan et al., 2009) and generalized to 3D rotationally invariant schemes (Lawrenz and Finsterbusch, 2013; Jespersen et al., 2013) and experimentally demonstrated in numerous systems ranging from porous media and phantoms to neural tissue ex- and in-vivo (Shemesh and Cohen, 2008, 2011; Jespersen et al., 2013; Shemesh et al., 2010; Shemesh et al., 2011; Koch and Finsterbusch, 2011; Lawrenz et al., 2015). The main advantage of such DDE approaches is that they can deliver the information on the microscopic anisotropy directly from only a few points along the angular curve, thereby providing an efficient way of inferring the underlying geometry. As well, they can differentiate between multicomponent Gaussian diffusion and non-Gaussian, restricted diffusion directly from the shape of the angular modulation.

The first spectroscopic DDE implementation utilizing the chemical shift to separate compartments and DDE filters to gain sensitivity towards microstructure was given in Shemesh et al. (2011) on an emulsion system mimicking a "cellular" and "extracellular" environment. The spectral dimension here was greatly simplified compared to in vivo spectra, and contained only two signals: the toluene (intra) and water (extra) signals in roughly equal amounts. SDE q-space experiments could not clearly differentiate the two signals, and in fact it was unclear whether they exhibit compartmentation at all. By contrast, the DDE MRS experiments (non-localized) showed clearly that there are two different diffusion behaviors: the first, representing diffusion within big spheres (toluene) and the second exhibiting restricted diffusion in a randomly oriented environment with a high eccentricity (water). These chemical-shift driven specificity enhancements, coupled with DDE's ability to resolve microscopic anisotropy unambiguously defined the total microstructure of a complex system, and thus provided strong incentives for in vivo DDE MRS experiments.

The first in vivo DDE MRS experiments were performed using the RE MRS approach specifically tailored for NAA, tCr, tCho, and Lactic acid (Lac) signals in a rat stroke model, where voxels were localized in ipsiand contra-lateral sides (Shemesh et al., 2014b). Raw data and results are then shown in Fig. 5B and C. DDE's characteristic modulation curves are easily evident in the raw signal stacked plots, and can be further appreciated from the plots. Several features were noted: (1) the metabolites unequivocally exhibit restricted, non-Gaussian diffusion, and the shape of the curve suggested their localization in highly eccentric compartments; (2) the stroke altered the geometry of the intraneuronal compartment within the voxel, as evident from the statistically significant difference in NAA's modulation between the hemispheres; (3) Lactic acid moves from a rather spherical compartment to a much more eccentric compartment upon ischemia.

A later DDE RE MRS study (Shemesh et al., 2015), this time performed in the normal rat brain, used a slightly different excitation/refocusing pulses, such that their bands encompassed only NAA and Ins signals, in an effort to discern between the neuronal and astrocytic compartments more clearly. As mentioned earlier, NAA is a specific intra-neuronal marker, while Ins is present nearly exclusively in the intra-astrocytic compartment. Fig. 6A shows how the multiband pulse indeed excited only Ins and NAA signals, greatly simplifying the spectrum (n.b., it is displayed in magnitude mode to avoid the deleterious effects of J-coupling and since the spectral resolution is not that 


\section{Cellular specific RE MRS}
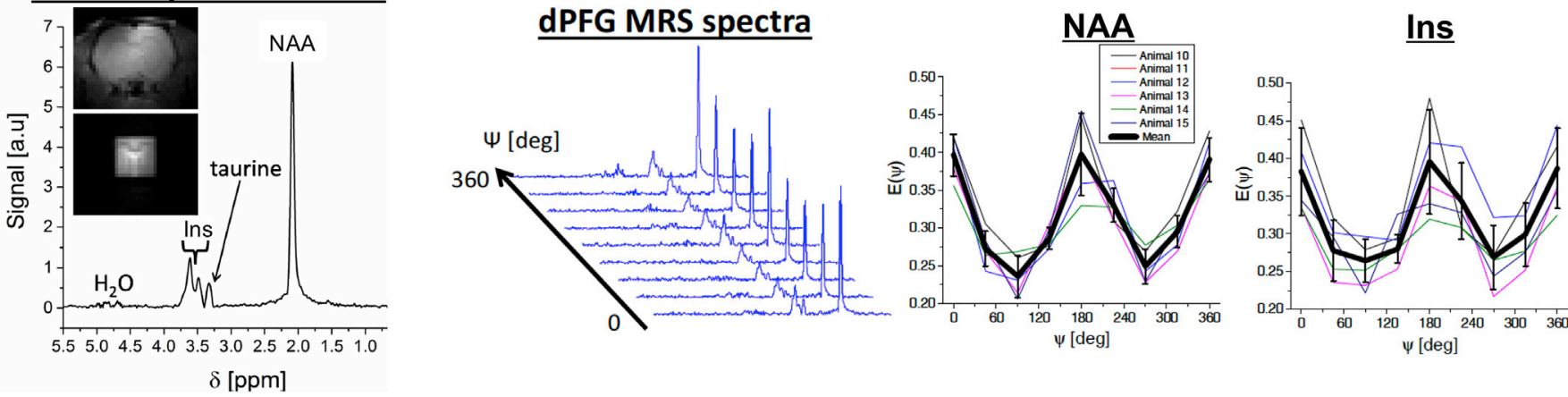

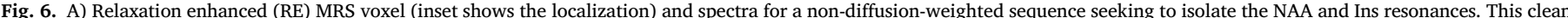

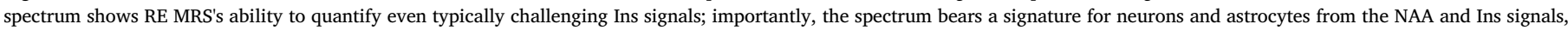

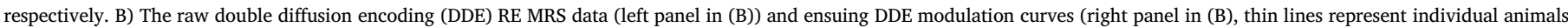

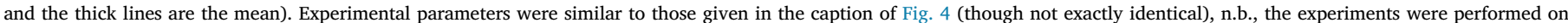

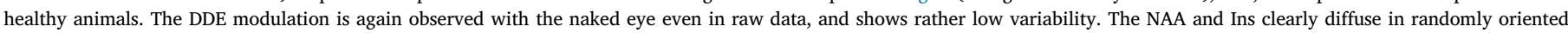

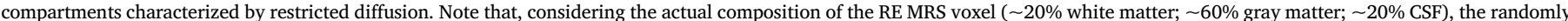

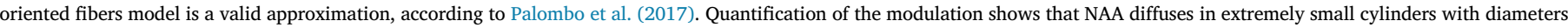
$<0.1 \mu \mathrm{m}$, while Ins diffuses in cylinders with diameter $\sim 3 \mu \mathrm{m}$ (Shemesh et al., 2015).

important due to the band-limited excitation), and facilitating the DDE acquisition. The clear DDE modulation observed (Fig. 6B) extends the previous findings, and supports the notion that NAA and Ins are restricted in elongated compartments.

To summarize, the measured characteristic oscillations for NAA, tCr, tCho and later for the Ins signals, can only be attributed to restricted diffusion (Figs. 5 and 6). Multi-Gaussian diffusion would not give rise to such modulated curves (Mitra, 1995). Thus, intracellular diffusion within neurons and astrocytes could be confirmed as non-gaussian from two independent techniques: time dependent SDE, and DDE. Another feature that can be compared between SDE and DDE experiments is the degree of anisotropy in each compartment. DDE's signal modulations revealed clear signatures for microscopic anisotropy, i.e., non-spherical components, with similar yet not identical microstructures (e.g., length/radius) for both neurons and astrocytes. A reasonable hypothesis for this observation is that these experiments represent diffusion in randomly oriented neurites in neurons, whereas in astrocytes, the compartments probed are the randomly oriented astrocytic processes. This is largely consistent with the conclusions drawn for observing ADC time-dependency over a very large range of diffusion times (Palombo et al., 2016).

\section{Estimating fiber diameter from the high $b / q$-value signal attenuation}

At intermediate $t_{d}$, i.e., between 10 and $100 \mathrm{~ms}$, the fraction of metabolites experiencing branching during their diffusion along a cell fiber process may be neglected and the cell fiber can be in good approximation modeled as a long infinite cylinder. Considering an average metabolite $\mathrm{D}_{\text {free }} \sim 0.4 \mu \mathrm{m}^{2} / \mathrm{ms}$ and an average fiber length between successive embranchments $\mathrm{L}_{\text {segment }} \sim 30 \mu \mathrm{m}$ (Palombo et al., 2016; Oberheim et al., 2009), for $t_{d} \leq 100 \mathrm{~ms}$ the mean metabolite displacement along the cell process is $\leq 10 \mu \mathrm{m}<<\mathrm{L}_{\text {segment. }}$ In this regime, cellular fibers can be modeled as long hollow cylinders. Because the spectroscopy voxel from which the DW-MRS signal is measured contains a large number of cellular processes randomly oriented in space, a model of randomly oriented cylinders in space has been proposed (Fig. 7A) to describe the observed non mono-exponential echo signal attenuation at high $\mathrm{q} / \mathrm{b}$ values (Yablonskiy and Sukstanskii, 2010; Kroenke et al., 2004; Palombo et al., 2017).

Assuming that the cylinder has a radius $a$, and the intracellular metabolites diffusivity is $\mathrm{D}_{\text {free }}$, the measured echo signal represents the sum of signals from a large number of differently oriented cylinders. For any given cylinder whose axis makes a variable angle $\theta$ with the diffusion gradient, the echo signal attenuation is described by Eq. (5). The total echo signal attenuation, $\mathrm{S}$, as measured from a large spectroscopy voxel is then given by:

$\frac{S\left(q, t_{d}\right)}{S\left(q=0, t_{d}\right)}=\frac{\int_{0}^{\pi / 2} p(\theta) \frac{S_{c y l i n d e r}^{\theta}\left(q, t_{d}\right)}{S\left(q=0, t_{d}\right)} d \theta}{\int_{0}^{\pi / 2} p(\theta) d \theta}$

where $p(\theta)=\sin (\theta)$ is the distribution of fibers making an angle $\theta$ relative to a fixed gradient direction in the case of an isotropic fiber distribution. If the fibers are expected to follow a specific orientation distribution (as in the case of a voxel entirely within white matter tracts) or a dispersion pattern, as for example in the DW-MRI NODDI model (Zhang et al., 2012), Eq. (6) can be opportunely modified in order to take into account the specific fiber orientation or dispersion. While fiber orientation and dispersion is important and has to be taken into account, for example, in DW-MRS experiments in human brain white matter (see Ronen et al. (2014) and following Section 3), within the large spectroscopy voxel in preclinical experiments on mouse brain, fibers can be assumed isotropically distributed with good approximation (see Supplementary Information in Palombo et al. (2017)).

A thorough investigation of NAA diffusion in brain cells at high $\mathrm{q} / \mathrm{b}$ values was done by Assaf and Cohen in the late 1990s (Assaf and Cohen, 1999, 1998a, 1998b). In these pioneering works (for a comprehensive review of early diffusion MRS studies we direct the reader to Nicolay et al. (2001)), Assaf and Cohen characterized the restricted diffusion behaviour of NAA by showing its bi- and tri-exponential diffusion decays within a large range of b-values (up to $35000 \mathrm{~s} / \mathrm{mm}^{2}$ ) and diffusion times (up to $300 \mathrm{~ms}$ ). Later on, Kroenke et al. and Yablonskiy and Sukstanskii (Yablonskiy and Sukstanskii, 2010; Kroenke et al., 2004) proposed a first attempt to model NAA diffusion taking into account cellular structure by proposing a model of randomly oriented cylinders, in which the radial diffusivity was set to 0 (i.e., randomly oriented sticks). In this case, the total echo signal decay differs from the one in Eq. (6), and is described by:

$\frac{S\left(q, t_{d}\right)}{S\left(q=0, t_{d}\right)}=\left(\frac{\pi}{4 A D C_{\text {axial }} q^{2} t_{d}}\right)^{1 / 2} \operatorname{erf}\left[\left(A D C_{\text {axial }} q^{2} t_{d}\right)^{1 / 2}\right]$

where $\mathrm{ADC}_{\text {axial }}$ is the metabolite axial diffusivity, the $\mathrm{ADC}_{\text {radial }}$ is set to 0 , and $\operatorname{erf}[\ldots]$ is the error function.

The choice to set the NAA radial diffusivity to 0 was based on the observation that the estimated radial diffusivity was an order of magnitude lower than the estimated axial one. However, the zero radial 
A

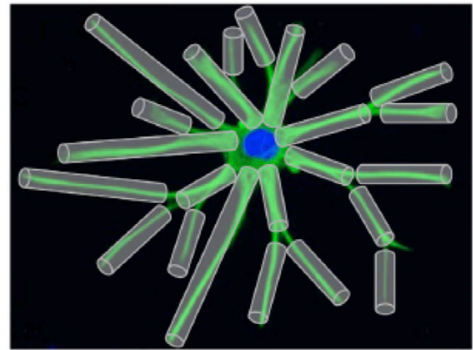

Randomly Oriented Cylinders Model
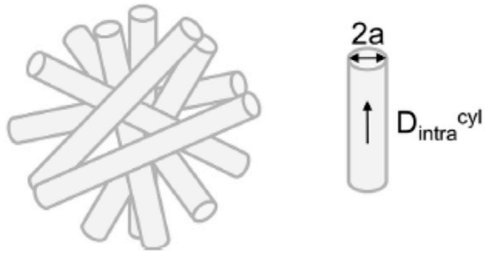

C

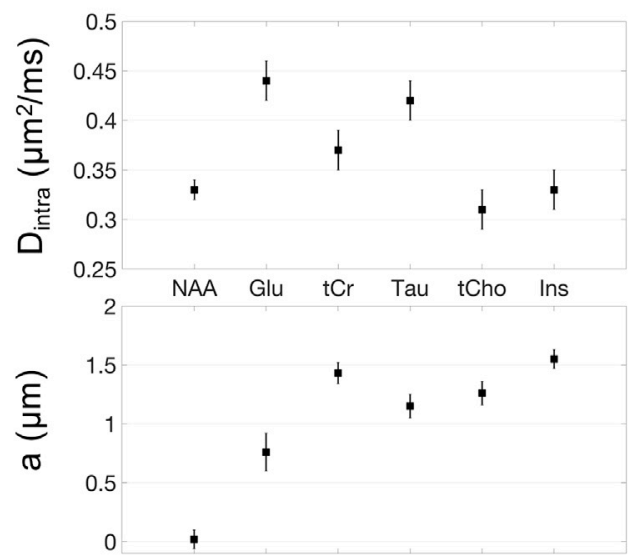

$\mathrm{B}$
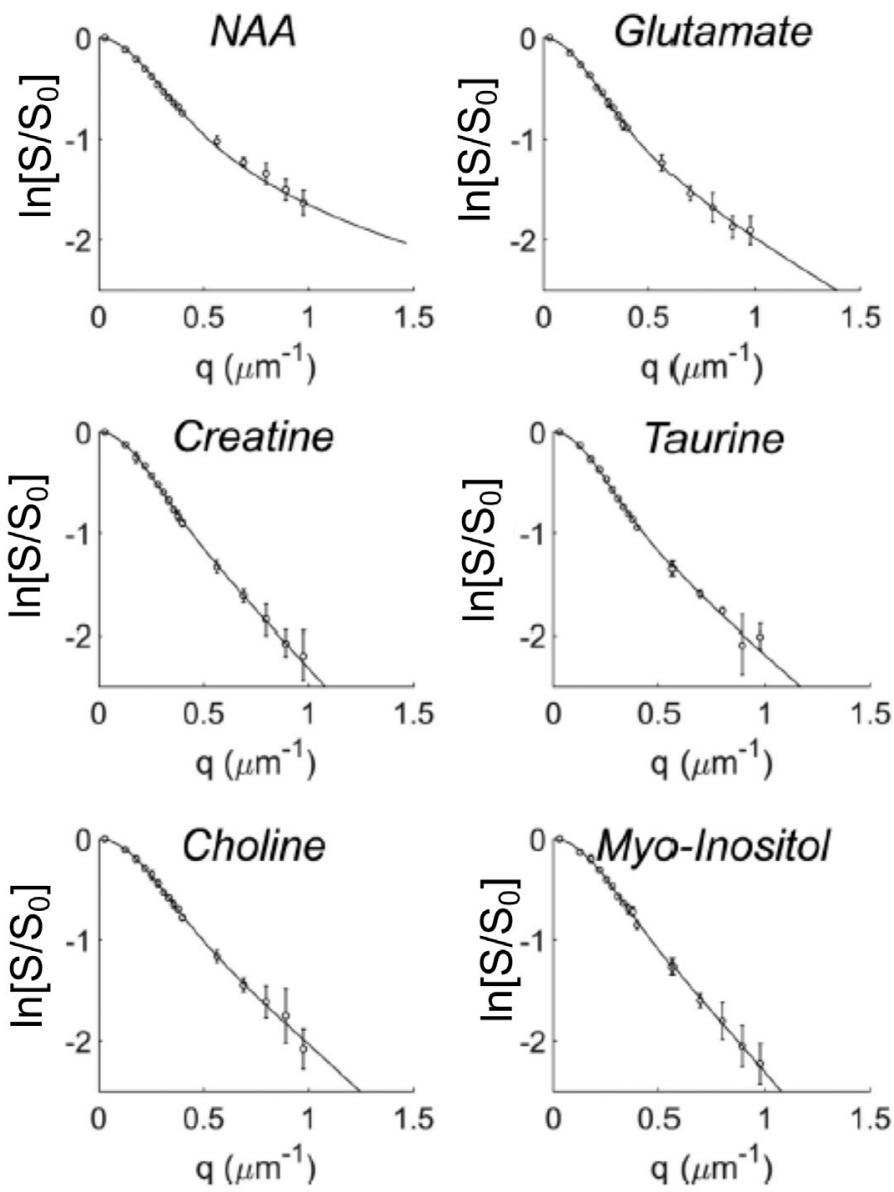

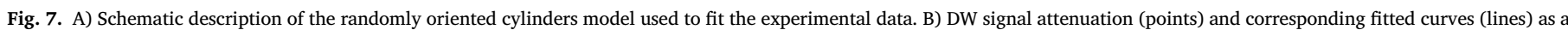

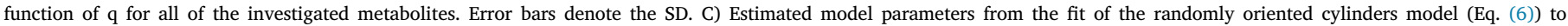
experimental data of each metabolite. Note: $\mathrm{D}_{\text {intra }}=$ intracellular diffusivity; $a$ = cylinder's radius (mean \pm SD, 2500 Monte Carlo draws). From Palombo et al. (2017).

diffusivity assumption should not be considered true in systems with large nerve fibers and at short diffusion times, as shown, for example, by DT-MRS experiments on frog sciatic nerve (Ellegood et al., 2007). In fact, in this kind of systems, Ellegood and coworkers showed a non-zero radial diffusivity for NAA, tCr, tCho, Taurine (Tau) and Glutamine-Glutamate-GABA complex (Glx) (e.g. $0.15-0.18 \mu \mathrm{m}^{2} / \mathrm{ms}$ ), for diffusion time of $18 \mathrm{~ms}$ (Ellegood et al., 2007).

Moreover, recent DW-MRS studies of other metabolites like Glu, tCr, tCho and Ins, in mouse brain showed that the assumption of zero-radius is not generally valid (Palombo et al., 2017). Palombo et al. recently used the model in Eq. (6) to characterize healthy in vivo mouse brain at $11.7 \mathrm{~T}$, showing that randomly oriented cylinders assumption accounts well for measured echo attenuation for Glu, tCr, Tau, tCho and Ins (Fig. 7B), yielding fiber radii and $\mathrm{D}_{\text {free }}$ in the expected ranges $(0.5-1.5 \mu \mathrm{m}$ and $0.30-0.45 \mu \mathrm{m}^{2} / \mathrm{ms}$, respectively, Fig. 7C) (Palombo et al., 2017). Interestingly, the only exception was NAA, for which the extracted radial diffusivity and radius was 0 . A small correction was proposed to the model and showed that the echo signal attenuation for NAA is compatible with a model where the majority of the NAA volume fraction diffuses in randomly oriented cylinders of non-zero radius, and a small fraction of the NAA $(\leq 10 \%)$ is instead confined in highly restricted compartments where the NAA does not diffuse and has a short $\mathrm{T}_{2}$ compared to the cytosolic NAA (Palombo et al., 2017). The authors suggested that this small fraction may be representative of the NAA confined in mitochondria, where NAA is synthesized, and within the myelin sheath of neuronal axons. The introduction of this immobile NAA pool allowed the estimation of reasonable values for $D_{\text {free }}$ and fiber radius $\left(\sim 0.34 \mu \mathrm{m}^{2} / \mathrm{ms}\right.$ and $\sim 0.6 \mu \mathrm{m}$, respectively), supporting the effectiveness of the model in Eq. (6). This fiber radius estimated from NAA diffusion was close to that estimated from Glu diffusion $(\sim 0.8 \mu \mathrm{m})$, while radii estimated from other metabolites appeared larger (the largest, $\sim 1.6 \mu \mathrm{m}$, being found for Ins), suggesting that these non-neuronal metabolites are experiencing less radial diffusion in glial cells.

It may be surprising that DW-MRS allows the estimation of so small fiber diameters $(<3 \mu \mathrm{m})$. Indeed, it is now well known that for water based DW-MRI, there is a lower limit to the sensitivity to fiber diameter which sets the minimum accessible diameter by using a single diffusion encoding sequence, $\mathrm{d}_{\mathrm{min}}^{(\mathrm{SDE})}$, at (Nilsson et al., 2017):

$d_{\min }^{(S D E)}=\left(\frac{768}{7} \frac{\sigma D_{\text {free }}}{\gamma^{2} \delta g^{2}}\right)^{1 / 4}$

where $\sigma$ is the normalized standard deviation of the signal due to noise. For example, for the typical clinical high-SNR case (SNR $=50$ ), where $\sigma=1 \%$, water $D_{\text {free }}=2 \mu \mathrm{m}^{2} / \mathrm{ms}, \mathrm{g}=80 \mathrm{mT} / \mathrm{m}$ and $\delta=40 \mathrm{~ms}$, $\mathrm{d}_{\min }^{(\mathrm{SDE})}=3.3 \mu \mathrm{m}$. In similar experimental conditions, for metabolites with an average $D_{\text {free }}=0.4 \mu \mathrm{m}^{2} / \mathrm{ms}$, it is possible to reach $\mathrm{d}_{\min }^{(\mathrm{SDE})}=2.2 \mu \mathrm{m}$. This significant improvement in diameter sensitivity is due to the much lower metabolites diffusivity compared to that of water molecules. Considering the specific preclinical experimental setup used in the high q/b values experiments reported in Ligneul et al. (2017), for NAA with $\mathrm{D}_{\text {free }}=0.34 \mu \mathrm{m}^{2} / \mathrm{ms}, \sigma=1 \%, g_{\max }=750 \mathrm{mT} / \mathrm{m}$ and $\delta=3 \mathrm{~ms}$, the 

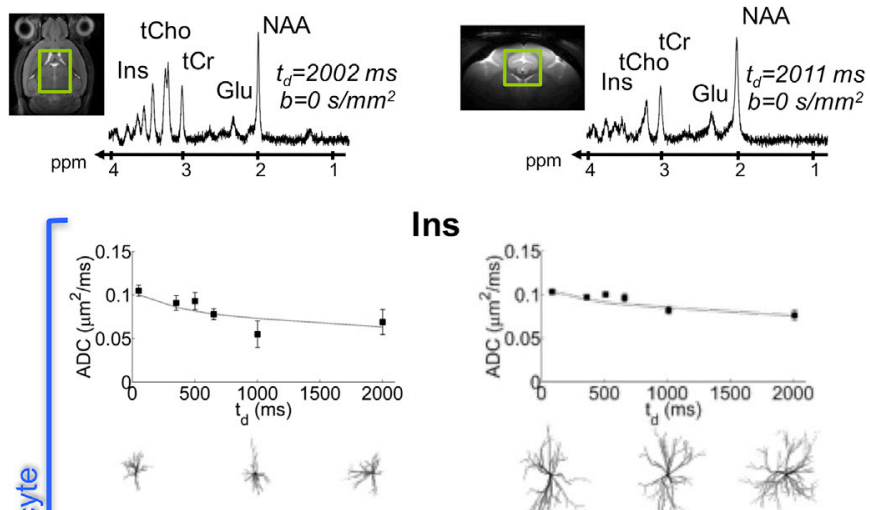

Ins

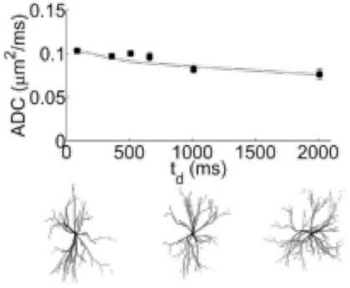

tCho
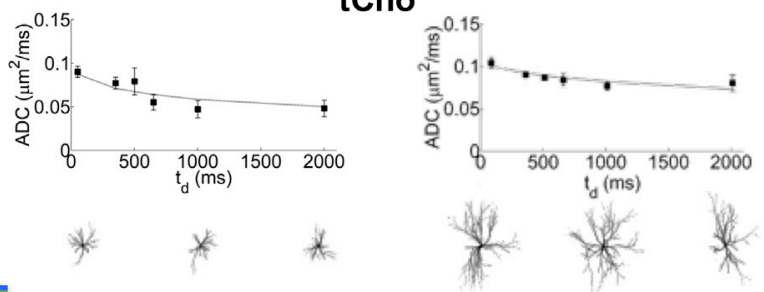

$\mathrm{tCr}$
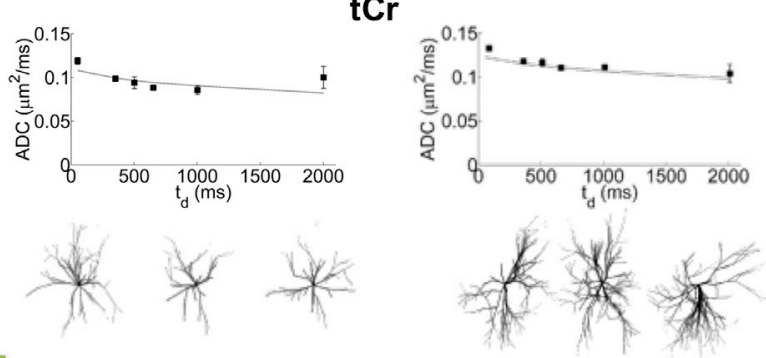

Glu
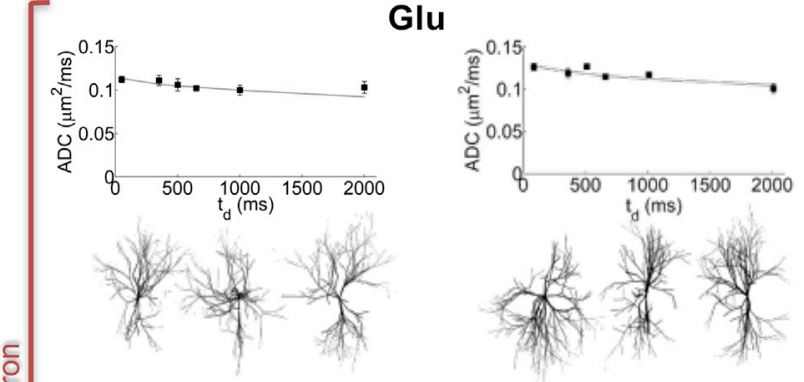

tNAA

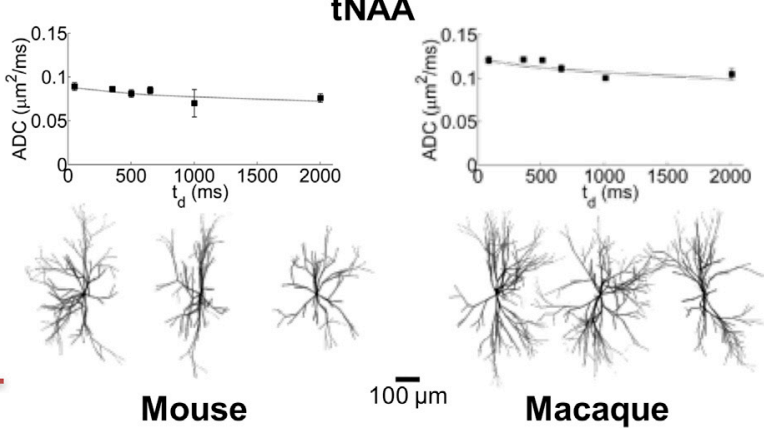

Fig. 8. DW-MRS results and modeling in the mouse and macaque brain. The investigated volume of interest within the brain (green box) and a typical DW-MRS spectrum at $t_{d}=2 \mathrm{~s}$ (and $\mathrm{b}=0 \mathrm{~s} / \mathrm{mm}^{2}$ ), as used to measure (using diffusion sensitizing gradients simultaneously applied along the three main axis $\mathrm{x}, \mathrm{y}$ and $\mathrm{z}$ ) ADC time dependence for each metabolite (Inset plots), are shown for each species. Points and error bars stand for ADC means and standard deviation of the means, respectively, estimated among the cohorts. resulting $\mathrm{d}_{\mathrm{min}}^{(\mathrm{SDE})}$ is $1.3 \mu \mathrm{m}$.

Experimental results and theoretical investigations reported in this session show very reasonable estimates for the brain cell fiber diameter. However, concerning fiber diameter estimation, one needs to be careful because there is still lack of experimental validations by direct histological measurements.

\section{Estimating fiber diameter from DDE RE MRS experiments}

Interestingly, the fiber diameter question for NAA and for Ins, representing the neuronal and astrocytic compartments of the CNS tissue, was tackled also from the DDE angle. The data in Shemesh et al. (2015), other than providing evidence for fitting a randomly oriented anisotropic compartment model, can also be used for fitting the compartment's diameter. To do so, a large multidimensional dictionary of signals was simulated in the MISST toolbox (Drobnjak et al., 2011; Ianus et al., 2016), with all sequence parameters (diffusion times, mixing times, gradient durations, gradient amplitudes, and number of measurements) input directly to the simulation. Based on the shape of the curve, a randomly oriented infinite cylinder model was chosen, with finely sampled diameters, and, importantly, the "free" diffusivity of the metabolite was also varied on a very fine grid for each diameter value. This provided a "fitting plane" ( $\left.D_{\text {free }}, d\right)$, where $D_{\text {free }}$ is free diffusivity and d the diameter, to which the data was regressed. When the DDE modulation curves shown in Fig. 6 are carefully quantified for NAA and Ins resonances, using this approach, the fiber diameter was found to be less than $1.3 \mu \mathrm{m}$ for NAA, and between 2 and $4 \mu \mathrm{m}$ for Ins, with the local minima approaching $0.1 \mu \mathrm{m}$ for NAA and $3.1 \mu \mathrm{m}$ for Ins. These results represent the average diameter of randomly oriented cylinders. These results are very much in line with those reported for the high $b / q$ values experiments described above.

To summarize, the two independent measurements - SDE high b/q values and angular DDE - provided similar results and reinforce each other. The NAA diffuses in fibers somewhat smaller than the astrocytes, and in both cases, for the experimental designs chosen, the sequences seem to be much more sensitive to the randomly oriented neurites or astrocyctic processes, respectively.

Cellular long-range microstructure: cell fiber segment length and number of embranchments

At long diffusion times ( $>100 \mathrm{~ms}$ ), while it is possible to discard the effect of cell fiber diameter, which can be assumed to be zero, as well as finer secondary structures and consequent ADC time-dependency, the branching of cell fibers comprising the neuronal dendritic trees or astrocytes processes cannot be neglected. The only study investigating this so far showed that cell fiber finite length and embranchments induce a specific ADC time dependence at (ultra-)long $t_{d}$ (Palombo et al., 2016). In a modeling framework that treats fibers as mono-dimensional branching objects "embedded" in a three-dimensional space and uses massive Monte-Carlo simulations, Palombo et al. predicted the effect of different morphometric statistics (i.e. the number of successive embranchments $\mathrm{N}_{\text {branch }}$ along each process, and the segment length $\mathrm{L}_{\text {segment }}$ for a given segment comprising a cellular fiber process) on the measured ADC time dependence. This general model was used to analyze data acquired up to $t_{d}=2 \mathrm{~s}$ in the healthy mouse (at $11.7 \mathrm{~T}$ ) and macaque (at $7 \mathrm{~T}$ ) brain in vivo (Fig. 8). The proposed modeling framework consistently classified cellular compartments, strongly supporting the generally accepted preferential compartmentalization of Ins and tCho inside astrocytes and of Glu and NAA in neurons, whereas some other metabolites such as tCr and

Best fit of ADC (averaged over the cohorts) is also displayed as a continuous curve. A subset of the extracted synthetic cells for each metabolite is also reported. (Scale bar, $100 \mu \mathrm{m})$. From Palombo et al. (2016). Note that, considering the actual composition of the RE MRS voxel ( $\sim 20 \%$ white matter; $\sim 60 \%$ gray matter; $\sim 20 \% \mathrm{CSF})$, the randomly oriented fibers model is a valid approximation, according to Palombo et al. (2017). 
Tau seem to have no preferential compartmentalization. In addition, extracted cell morphologies, such as length of branch segments and number of embranchments, were qualitatively and quantitatively consistent with histological data (Fig. 9), suggesting that the effect of cell fiber length and embranchments must be considered when modeling long $t_{d}$ data.

\section{The use of mutual information from DWI and DW-MRS}

The task of extracting unequivocal microstructural information from diffusion weighted MR experiments is challenging, both in the case of DW-MRI and that of DW-MRS. In DW-MRI it is the lack of compartmental and cellular specificity and inter-compartmental exchange that pose the major challenge, as the sensitivity is high enough to allow reasonably low partial volume across white matter tracts, as well as enough "pure" gray and white matter voxels for tissue specific analysis. Conversely, in DWMRS the major challenge stems from the intrinsic low sensitivity of the method, while the specificity, at least for some of the most prominent metabolites, is high. A natural consequence would thus be combining DW-MRI and DW-MRS in an analysis framework that benefits from the complementarity of the two methods. Few works so far have attempted to propose such a framework, and here we will briefly survey what has been done so far and what are some of the possible directions in which coanalysis of DW-MRI and DW-MRS data can evolve to provide a more comprehensive picture of tissue microstructure.
How can DW-MRS inform DW-MRI?

Insights from separate DW-MRS measurements that can have a deep impact on e.g. modeling of DW-MRI data have been already previously mentioned, e.g. the results from DW-MRS experiments in both animals and in humans that show no evidence for the existence of fully restricted compartments in neural tissue, up to diffusion times in the order of 1-2 s (Najac et al., 2014, 2016). Another DW-MRS result with implications on the way DW-MRI data should be interpreted is that the ADC values of all metabolites in gray matter are consistently lower than in white matter (i.e. see Table 1 or (Kan et al., 2012; Branzoli et al., 2014; Ercan et al., 2015)) as opposed to the ADC of water, which is homogeneous across brain tissue when measured in the typical range of $b \approx 1000 \mathrm{~s} / \mathrm{mm}^{2}$, and becomes higher in GM for higher values of $b$ (Yoshiura et al., 2001). This may indicate that either the extracellular space in cortical gray matter is more loosely packed than in white matter, or/and that there are intrinsic differences in the viscosity of these tissues, or/and that cross-membrane water exchange between the intra- and extracellular space is faster. The latter hypothesis obtains some support from recent experiments, including a measurement of apparent exchange rate (AXR) using the filter exchange imaging (FEXI) technique (Nilsson et al., 2013). So far, few studies provide separate metabolite ADC data for gray and white matter, and more evidence is needed to fully characterize metabolite $\mathrm{ADC}$ in white matter and cortical/subcortical gray matter, both in humans and in animals. A recent DW-MRS study in multiple sclerosis

\section{A}
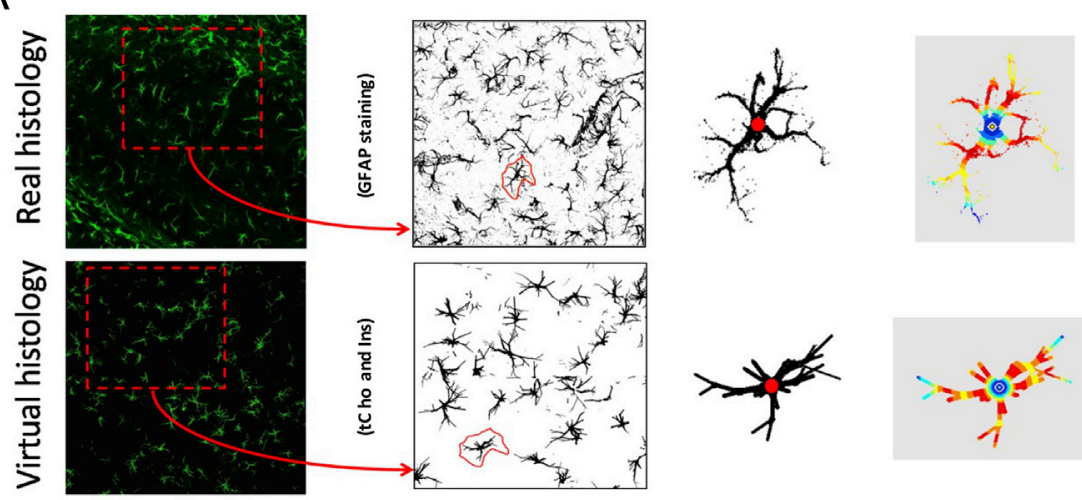

Zoom and binarization of bitmap images

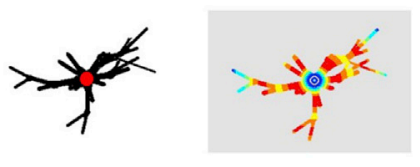

Single cell isolation and Sholl analysis

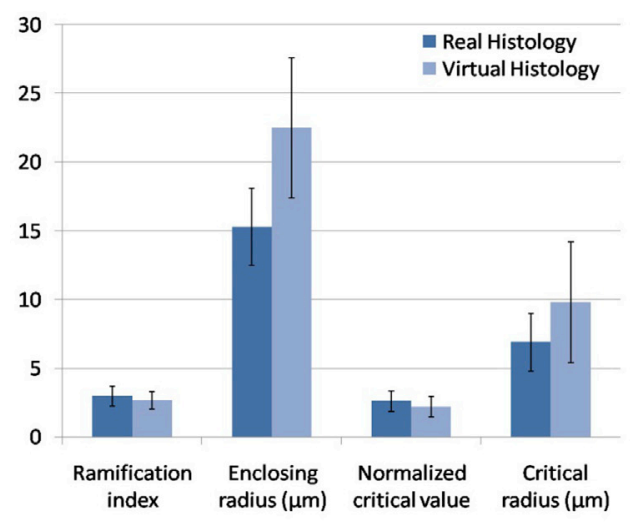

Estimation of Sholl-based metrics and comparison

B
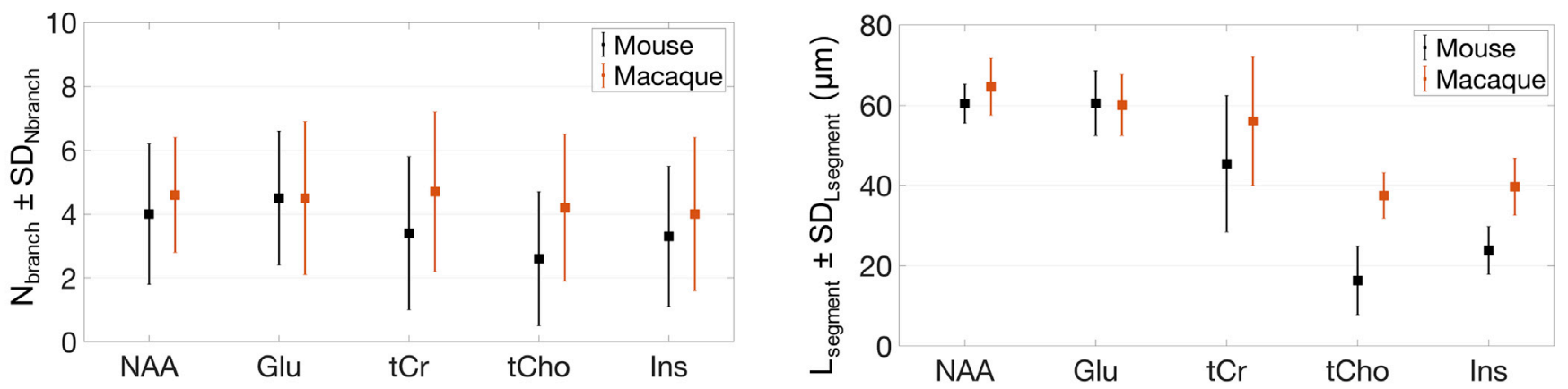

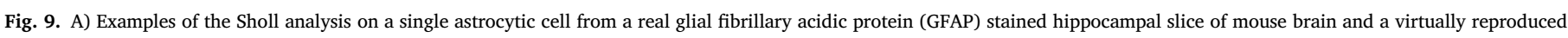

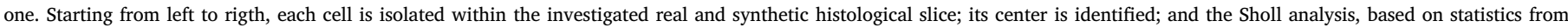

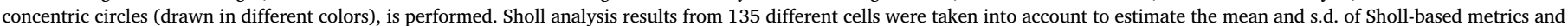

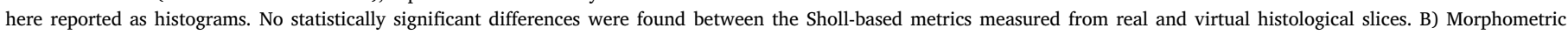

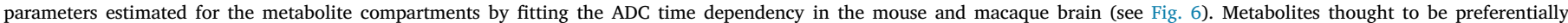

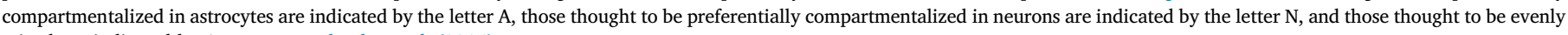
mixed are indicated by A+N. From Palombo et al. (2016). 
found that in the thalamus, for example, the ADC of NAA is higher than in parietal white matter (Bodini et al., 2017), indicating yet another possible set of intracellular conditions that lead to higher diffusivity in that particular region.

To envision the potential benefits of simultaneous use of mutual information from DW-MRI and DW-MRS, it is useful to look at an early example of DW-MRS in animal model of stroke (Wick et al., 1995). In this work, as well as in subsequent works (van der Toorn et al., 1996) a decrease in all metabolite ADC was measured in the acute ischemic phase. This is an independent evidence that supports the notion that the decrease in ADC in acute stroke is not solely a result of increased restriction of the extracellular space following intracellular edema (Budde and Frank, 2010). Additional support to these findings was provided by diffusion measurements of metabolites and water in brain slices (Hakumaki et al., 2000) as well as in humans (Harada et al., 2002).

Another example is that examines diffusion tensor metrics of the intraneuronal metabolite, NAA, and those of water in the same two volumes of interest in the human corpus callosum (CC) (Upadhyay et al., 2007). From the microstructural investigation stand point, the CC offers the simplest test-case possible: a single, easily identifiable white matter tract with a known orientation, which is mostly left-right in its medial part and then curves towards superior cortical regions. This structural simplicity led to the first work that modeled the diffusion of NAA in human white matter (Kroenke et al., 2004) and to the first reported diffusion tensor of metabolites from white matter regions (Ellegood et al., 2006). In Upadhyay et al. (2007), the diffusion tensors of both water and NAA are estimated from a DW-MRS experiment in which the water was suppressed for the spectroscopic data and left untouched for the water data. An estimation of the partial volume effect of CSF was essential for a more accurate estimation of the water tensor, but is not necessary for the estimation of the NAA tensor, as there is no NAA in the CSF. The FA values reported for NAA from the two callosal volumes were 0.72 and 0.52 for the anterior and more posterior VOI, respectively, and those of water were 0.46 and 0.39 . It should be emphasized that the FA of water as calculated from the DW-MRS VOI is significantly lower than the one that could be obtained from a DTI experiment in the medial part of the CC. This is due to the macroscopic curvature of the callosal fibers within the spectroscopic VOI, which in this case similarly affects the NAA and the water FA. The fact that the water FA values are lower is not surprising, since in addition to the microscopically and macroscopically highly anisotropic intra-axonal space unique to the NAA, water is also present in other cellular structures, e.g. glia, as well as in the extracellular space and in the myelin sheath. Assuming that the contribution of the latter is negligible at the long TE in which the experiments were performed (MacKay et al., 1994), it is theoretically possible to estimate, based on prior estimates of the intra-axonal and extra-axonal volume fractions in the VOIs, the FA of the extra-axonal compartment. The structural properties of the extra-axonal space in white matter are key in any modeling framework for DW-MRI data, and it is thus important to assess the validity of assumptions regarding its contribution to anisotropy, as these may greatly differ. This is indeed the case for the modeling framework CHARMED (Assaf and Basser, 2005) and NODDI (Zhang et al., 2012), where in CHARMED, as well as in other modeling frameworks (Jespersen et al., 2007; Behrens et al., 2003) the extra-axonal contribution to FA is independent of the intra-axonal contribution, whereas in NODDI the two contributions to FA are interdependent. It is important to note that when calculating FA values from DW-MRS experiments, noise propagation has to be carefully assessed, as FA values can become artificially high as a result of noisy measurements (Ellegood et al., 2011).

Another question regarding compartmental contributions to anisotropy is what is the volume fraction of the macroscopically isotropic compartment in tissue. It is safe to assume that a significant contribution to isotropic diffusion in tissue comes from structures that are on average isotropic. In neural tissue these can be glial cells such as astrocytes and microglia, although oligodendrocytes and fibrous astrocytes tend to align themselves to the white matter scaffold (Lundgaard et al., 2014) and thus probably preserving some degree of overall anisotropy. DW-MRS can be key in answering this question. In the first study that reported on tensor DW-MRS measurements, the overall fractional anisotropy of metabolites measured in VOIs that ranged between 10 and $12 \mathrm{cc}$ was similar across metabolites, also in white matter regions with one predominant white matter tract (Ellegood et al., 2006). A more recent study utilized the increased sensitivity of DW-MRS at 7T, and examined the diffusion of the three main metabolites in the corpus callosum in a small VOI $(2 \mathrm{cc})$ with b values up to $11,000 \mathrm{~s} / \mathrm{mm}^{2}$. In this work, the ratio between the diffusivity parallel and perpendicular to the callosal fibers within a DW-MRS VOI, $\frac{A D C_{\text {par }}}{A D C_{\text {perp }}}$, was almost twice as high for NAA compared to tCho and $\mathrm{tCr}$ (Ronen et al., 2013). Based on this finding, assuming that in white matter NAA is exclusively contained in axons, and that these are the sole source of intracellular macroscopic FA in the volume, it is possible to estimate the fractional volume of the isotropic compartments that contain the majority of tCho and tCr, based on their values of $\frac{A D C_{p a r}}{A D C_{p e r p}}$ and their overall tissue concentration. Based on the assumptions made above, it was estimated that the glial fraction of tCho is 0.5 and that of $\mathrm{tCr}$ is 0.4 (Ronen et al., 2013). Although this estimate does not take into account the volume fraction of axons and glia in white matter, it supports the notion that tCho is highly present in glia, more so than e.g. tCr. Stronger diffusion anisotropy of NAA compared to $\mathrm{tCr}$ and tCho was also reported in the bovine optic nerve for the so-called "fast ADC" derived from a biexponential analysis (Assaf and Cohen, 1999), whereas in a peripheral nerve DW-MRS measurement, the FA of NAA and Glu, both neuronal metabolites, resulted lower than that of tCr and tCho (Ellegood et al., 2007). These diverse results call for more DW-MRS studies in different systems in vivo as well as in vitro. A similar ratio to the one reported in Ronen et al. (2013) of $\mathrm{ADC}_{\mathrm{par}} / \mathrm{ADC}_{\text {perp }}$ for NAA in the corpus callosum was reported earlier (Kroenke et al., 2004). This work, however, did not report findings from other metabolites.

\section{How can DW-MRI inform DW-MRS?}

Keeping in mind that the main limitation of DW-MRS is its limited sensitivity, reflected in a significantly lower SNR compared to DW-MRI, it is inevitable that the spatial resolution of DW-MRS would be much lower. To compensate for 3-4 orders of magnitude in concentration for most of the proton resonances of interest in the MR spectrum, a concomitant increase in volume is needed, as shown in the example of Fig. 10. In standard MRI scanners this necessitates VOI of a few milliliters, thus with single dimensions in the order of centimeters. As a consequence, volumes of moderate size in white matter will include a broad axonal angular distribution, stemming both from the orientation dispersion across fibers and from the macroscopic factors such as the curvature of the fibers propagating within the VOI and multiple white matter tracts passing through the VOI (Fig. 10). In the case of e.g. arbitrary VOI position in parietal white matter this results in an almost uniform directional distribution, as confirmed by examining the angular distribution of the principal eigenvectors (Branzoli et al., 2014). Even when the VOI is significantly smaller and positioned on a single tract such as the CC, the contribution of the macroscopic curvature to the orientational distribution within the VOI is significant (Ronen et al, 2013, 2014). Since the curvature and shape of the CC within the VOI may significantly vary across subjects, this macroscopic angular distribution is a source of unwanted variance to DW-MRS measurements that can obfuscate e.g. differences between metabolite diffusion properties across subject populations in studies that examine the diffusivity of NAA as a marker for axonal degeneration (Wood et al., 2012; Du et al., 2013).

As seen in Section 2.3, Eq. (6), for the diffusion of NAA in white matter, a way to account for this confound is to model the data assuming diffusion in a set of cylinders with a given angular distribution with respect to the gradient direction. The angular distribution generated by the macroscopic curvature of the tract can be obtained from a separately performed DTI experiment, from which the set of $E_{1}$, the principal 

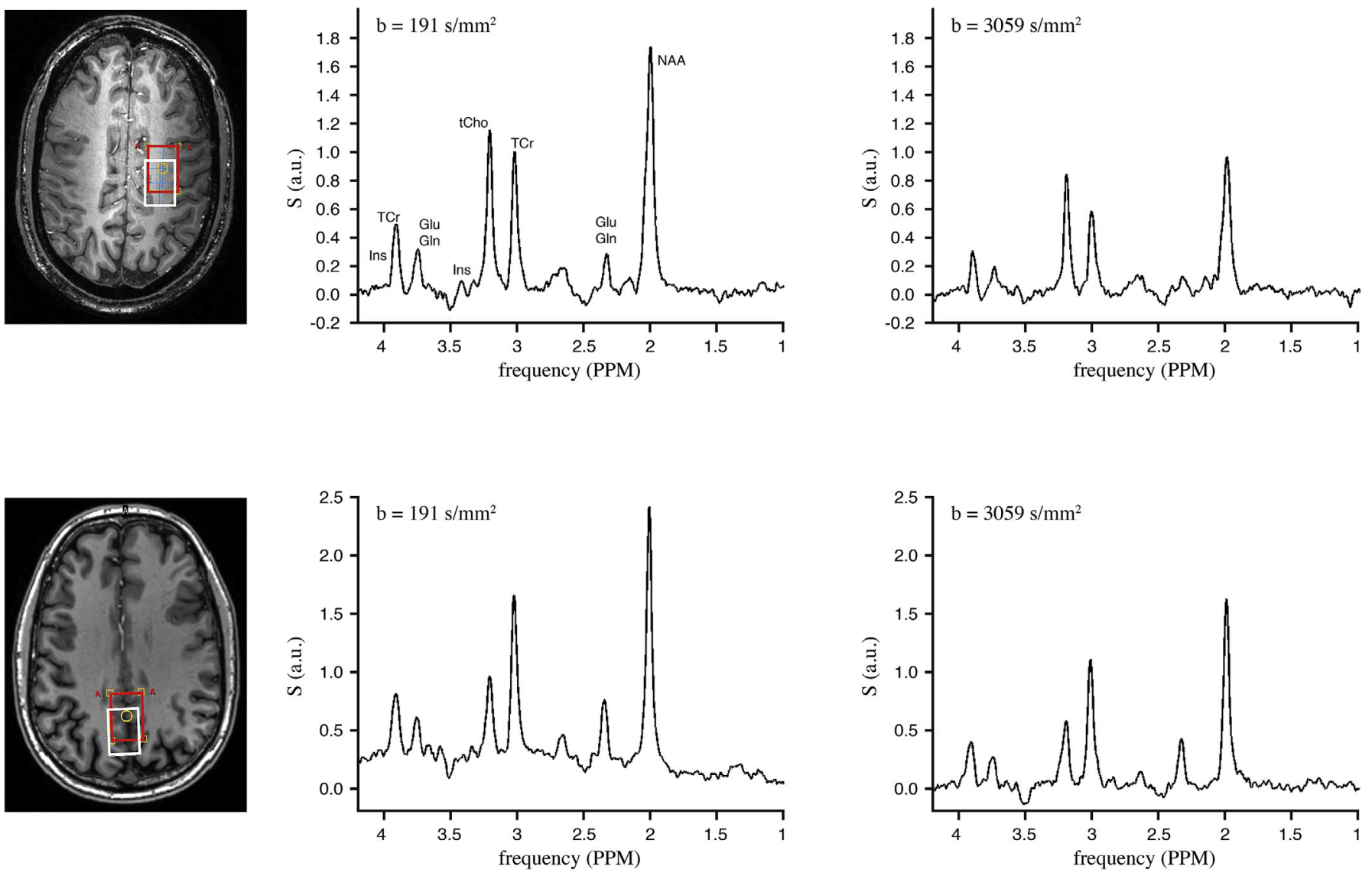

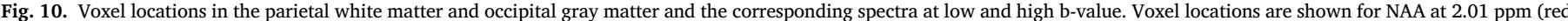

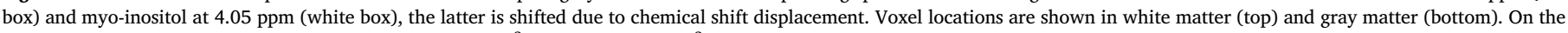

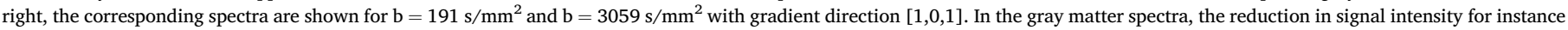
NAA and Glu and Gln is clearly reduced compared with white matter.

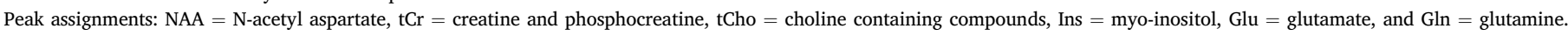
Spectra are shown with $2 \mathrm{~Hz}$ line broadening.

eigenvectors of the diffusion tensors of voxels within the spectroscopic VOI can be extracted (Ronen et al., 2014). This process is illustrated in stream A of Fig. 11.

In an additional step, it is possible to include an additional, microscopic distribution in convolution with the macroscopic one, to account for axonal orientation dispersion (stream B in Fig. 11). This results in a two-parameter model that not only accounts for the macroscopic confound, but also delivers an estimate for the orientation dispersion, shown to realistically fit with estimates from histology.

When DW-MRS is co-analyzed with DWI data, it is crucial to take into account the fact that actual localization of the various metabolites in the VOI is different due to frequency-dependent displacement of the VOI. This calls for accurate estimation of the real localization for each metabolite, especially for sequences such as PRESS, where the low bandwidth of the refocusing pulses can cause significant displacement along their slab selection axes.

Non-biased measurements of e.g. microscopic anisotropy of neuronal and glial processes can be also achieved directly from DW-MRS without using DTI data. This can be done by either the use of DDE experiments, as discussed in Section 2.3, or by generating the so-called "powder average" of multidirectional DW-MRS data at multiple diffusion weighting values, assuming that the sole source of deviation from monoexponential decay is the orientation dispersion (Lundell et al., 2017). These methods are not mutually exclusive to each other, and at this stage cross validation of methods and their assessment with respect to stability, reproducibility and time consumption is essential.
Clinical implications and future directions in DW-MRS methodology

\section{Multivoxel DW-MRS and DW-MRSI}

Work that has been done so far in disease implies that not only DWMRS is possible to apply in clinical settings, but that the potential of added information to that obtained from DWI experiments justifies the effort. So far, DW-MRS has been applied to study cerebral ischemia in animal models (van der Toorn et al., 1996; Wick et al., 1995; Abe et al., 2000) and in humans (Harada et al., 2002; Zheng et al., 2012), cerebral tumors in animals (Valette et al., 2012) and humans (Harada et al., 2002), normal aging (Zheng et al., 2012; Branzoli et al., 2016), schizophrenia (Du et al., 2013) and several studies in MS (Wood et al., 2012, 2017; Bodini et al., 2017). Although valuable microstructural information can be obtained from combining simple DTI data and DW-MRS data acquired from a single volume, a significant effort should be invested in generating robust multivoxel DW-MRS data, as this will be essential for a spatially-resolved combination of data from the two modalities with significant gains for tissue microstructural characterization in healthy and more importantly for diseased conditions. As was previously mentioned, the cytosolic diffusion coefficient of NAA is a good candidate for a putative marker for intracellular damage such as axonopathy in MS, but this role can be extended to other neurodegenerative disorders, such as tauopathy in $\mathrm{AD}$, where intraneuronal pathological changes are caused by hyperphosphorylation of the tau protein, which is responsible for keeping the integrity of microtubules (Ballatore et al., 2007; Delacourte and Buee, 2000; Grundke-Iqbal et al., 1986). In a combined DTI DW-MRS study of patients with neuropsychiatric systemic lupus 


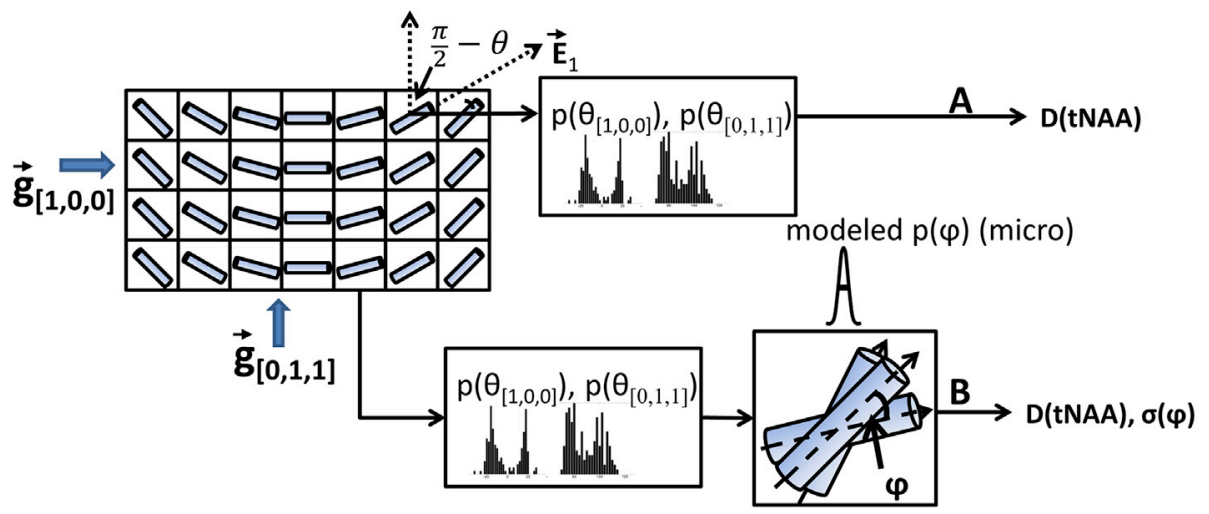

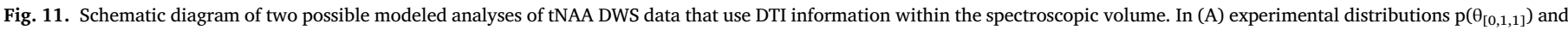

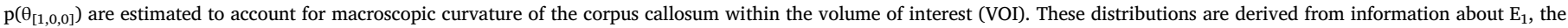

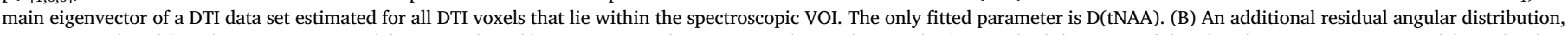

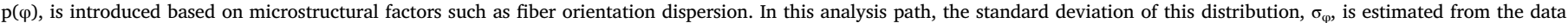
fitting procedure in addition to D(tNAA). From Ronen et al. (2014).

erythematosus (NPSLE), an increase in the diffusion coefficient of tCho was observed in correlation with neuropsychiatric symptoms and with the SLE disease activity index (SLEDAI), which scales with inflammatory state (Ercan et al., 2016). This points to the possibility that tCho's ADC is modulated by inflammation and reflects glial reactivity in response to inflammation. Reactive glia are known to undergo cytomorphological changes during activation, in line with increase in the ADC of intraglial metabolites (Verkhratsky et al., 2014). In this study, as well as in the DW-MRS study in multiple sclerosis., the DW-MRS findings correlated with DTI changes, and thus can mutually contribute to a better explanation of the disease process. The ability to do so in a spatially encoded manner over a large field of view is thus particularly attractive, as it offers the possibility to study water and metabolite diffusion in normal and abnormal appearing tissue, and map deficits across regions that may or may not correlate with disease outcome, together with e.g. PET tracers specific to cellular pathology.

A few efforts in developing sequences for DW-MRS in spectroscopic imaging mode have been published, both using conventional sequential k-space coverage, as well as using echo-planar spectroscopic imaging (EPSI) (Ercan et al., 2015; Bito et al., 1995, 2015; Fotso et al., 2017). Conventional acquisition of magnetic resonance spectroscopic imaging (MRSI) data is long and prone to errors that stem from phase and amplitude fluctuations. While phase variations can be accounted for in the post-processing stage, amplitude fluctuations and signal drop-outs should be dealt with prospectively. Approaches that use navigators, i.e. short time-domain signal acquired after the diffusion encoding but prior to the spatial encoding, have been proposed and implemented on clinical scanners (Fotso et al., 2017; Ercan et al., 2015), but robustness and sensitivity to disease effects has yet to be demonstrated. Efforts to shorten acquisition time would thus benefit from the use of parallel imaging (Bonekamp et al., 2010), and if SNR is high enough, perhaps a degree of compressed sensing (Cao et al., 2016; Cao and Wu, 2015).

DTI can also inform DW-MRS experiments in the planning phase, in cases where the diffusion properties of metabolites are to be studied e.g. along a particular white matter tract. In that case, DTI can be used to identify the tract of interest, and the DW-MRS can be subsequently performed on the desired tract, as was performed on the straight segment of the arcuate fasciculus, a tract involved in language processing (Upadhyay et al., 2008). The selected volume can also be spatially encoded with the application of phase encoding gradient in one dimension (1D-MRSI). This method has been shown to yield the diffusion tensor of NAA in several voxels along the straight portion of the corpus callosum (Wijnen et al., 2014) in good geometric agreement with the diffusion tensors of water data from the same volumes.
Additional strategies have been proposed for multivoxel DW-MRS that may offer simple and robust solutions with faster acquisitions, at the expense of less than full coverage of the brain. One approach is based on simultaneous acquisition of e.g. two separate volumes, which can be separated in the post-processing stage using information about coil sensitivities (Boer et al., 2015; Oeltzschner et al., 2017). This approach is similar to the multiband approach in imaging, e.g. Feinberg and Setsompop (2013). A dual volume acquisition approach can be useful in simultaneously acquiring data from two regions where one is visibly more affected by disease than the other, as in e.g. stroke or tumor that differentially affects one hemisphere.

\section{Conclusion}

In this review article, we surveyed the state-of-the-art methods that have been developed for robust acquisition, quantification and analysis of DW-MRS data, and discussed the potential relevance of DW-MRS for elucidating brain microstructure in vivo. There is still much to be done to further develop DW-MRS and bring it to a broader audience, both in terms of acquisition methodology as well as data analysis and modeling. The ever-improving hardware - better and stronger gradient systems, more sensitive RF coils, and higher static magnetic fields - feeds the hope that DW-MRS will become increasingly more useful to the scientific community. Some encouraging examples were reported and discussed, showing that with accurate data on diffusion of increasing number of metabolites, and with accurate computational and geometrical modeling, metabolite DW-MRS can provide unique cell-specific information on the intracellular structure of brain tissue. Since the implementation of an imaging version of DW-MRS is still in its infancy, the integration of mutually compatible information derived from a combined DW-MRI and DW-MRS approach seems to be, at the moment, a more practicable route towards a better cell-specific characterization of brain microstructure.

\section{Acknowledgements}

MP is grateful for funding from The Engineering and Physical Sciences Research Council grant code: N018702. NS is grateful for funding from the European Research Council under the European Union's Horizon 2020 research and innovation program (grant agreement No. 679058 - DIRECT-fMRI), as well as under the Marie Sklodowska-Curie grant agreement No 657366. JV is recipient of grant from the European Research Council about diffusion-weighted MRS (grant agreement No. 336331 - INCELL). 


\section{References}

Abe, O., et al., 2000. Temporal changes of the apparent diffusion coefficients of water and metabolites in rats with hemispheric infarction: experimental study of transhemispheric diaschisis in the contralateral hemisphere at 7 tesla. J. Cereb. Blood Flow. Metab. 20 (4), 726-735.

Adalid, V., et al., 2017. Fitting interrelated datasets: metabolite diffusion and general lineshapes. MAGMA 1-20.

Allison, S., Chen, C.Y., Stigter, D., 2001. The length dependence of translational diffusion, free solution electrophoretic mobility, and electrophoretic tether force of rigid rodlike model duplex DNA. Biophys. J. 81 (5), 2558-2568.

Assaf, Y., Basser, P.J., 2005. Composite hindered and restricted model of diffusion (CHARMED) MR imaging of the human brain. Neuroimage 27 (1), 48-58.

Assaf, Y., Cohen, Y., 1998. In vivo and in vitro bi-exponential diffusion of N-acetyl aspartate (NAA) in rat brain: a potential structural probe? Nmr Biomed. 11 (2), $67-74$.

Assaf, Y., Cohen, Y., 1998. Non-mono-exponential attenuation of water and N-acetyl aspartate signals due to diffusion in brain tissue. J. Magn. Reson 131 (1), 69-85.

Assaf, Y., Cohen, Y., 1999. Structural information in neuronal tissue as revealed by qspace diffusion NMR spectroscopy of metabolites in bovine optic nerve. Nmr Biomed. 12 (6), 335-344.

Avram, L., Assaf, Y., Cohen, Y., 2004. The effect of rotational angle and experimental parameters on the diffraction patterns and micro-structural information obtained from q-space diffusion NMR: implication for diffusion in white matter fibers. J. Magn. Reson 169 (1), 30-38.

Balinov, B., Jonsson, B., Linse, P., Söderman, O., 1993. The NMR self-diffusion method applied to restricted diffusion. Simulation of echo attenuation from molecules in spheres and between planes. J. Magn. Reson Ser. A 104, 17-25.

Ballatore, C., Lee, V.M., Trojanowski, J.Q., 2007. Tau-mediated neurodegeneration in Alzheimer's disease and related disorders. Nat. Rev. Neurosci. 8 (9), 663-672.

Bar-Shir, A., Avram, L., Ozarslan, E., Basser, P.J., Cohen, Y., 2008. The effect of the diffusion time and pulse gradient duration ratio on the diffraction pattern and the structural information estimated from q-space diffusion MR: experiments and simulations. J. Magn. Reson 194 (2), 230-236.

Basser, P.J., 1995. Inferring microstructural features and the physiological state of tissues from diffusion-weighted images. Nmr Biomed. 8 (7-8), 333-344.

Basser, P.J., Jones, D.K., 2002. Diffusion-tensor MRI: theory, experimental design and data analysis - a technical review. Nmr Biomed. 15 (7-8), 456-467.

Beard, E., Braissant, O., 2010. Synthesis and transport of creatine in the CNS: importance for cerebral functions. J. Neurochem. 115 (2), 297-313.

Behar, K.L., Ogino, T., 1993. Characterization of macromolecule resonances in the $1 \mathrm{H}$ NMR spectrum of rat brain. Magn. Reson Med. 30 (1), 38-44.

Behar, K.L., Rothman, D.L., Spencer, D.D., Petroff, O.A., 1994. Analysis of macromolecule resonances in 1H NMR spectra of human brain. Magn. Reson Med. 32 (3), 294-302.

Behrens, T.E., et al., 2003. Characterization and propagation of uncertainty in diffusionweighted MR imaging. Magn. Reson Med. 50 (5), 1077-1088.

Le Belle, J.E., et al., 2002. A comparison of cell and tissue extraction techniques using high-resolution 1H-NMR spectroscopy. NMR Biomed. 15 (1), 37-44.

Bhagat, Y.A., Beaulieu, C., 2004. Diffusion anisotropy in subcortical white matter and cortical gray matter: changes with aging and the role of CSF-suppression. J. Magn. Reson Imaging 20 (2), 216-227.

Le Bihan, D., et al., 2001. Diffusion tensor imaging: concepts and applications. J. Magn. Reson Imaging 13 (4), 534-546.

Bito, Y., Hirata, S., Nabeshima, T., Yamamoto, E., 1995. Echo-planar diffusion spectroscopic imaging. Magn. Reson Med. 33 (1), 69-73.

Bito, Y., et al., 2015. Diffusion-weighted line-scan echo-planar spectroscopic imaging technique to reduce motion artifacts in metabolite diffusion imaging. Magn. Reson Med. Sci. 14 (1), 43-50.

Bodini, B., et al., 2017. Dysregulation of energy metabolism in multiple sclerosis measured in vivo with diffusion-weighted spectroscopy. Mult. Scler. 1352458517698249.

Boer, V.O., Klomp, D.W., Laterra, J., Barker, P.B., 2015. Parallel reconstruction in accelerated multivoxel MR spectroscopy. Magn. Reson Med. 74 (3), 599-606.

Bonekamp, D., Smith, M.A., Zhu, H., Barker, P.B., 2010. Quantitative SENSE-MRSI of the human brain. Magn. Reson Imaging 28 (3), 305-313.

Bottomley, P.A., 1987. Spatial localization in NMR spectroscopy in vivo. Ann. N. Y. Acad. Sci. 508, 333-348.

Brand, A., Richter-Landsberg, C., Leibfritz, D., 1993. Multinuclear NMR studies on the energy metabolism of glial and neuronal cells. Dev. Neurosci. 15 (3-5), 289-298.

Branzoli, F., Techawiboonwong, A., Kan, H., Webb, A., Ronen, I., 2013. Functional diffusion-weighted magnetic resonance spectroscopy of the human primary visual cortex at 7 T. Magn. Reson Med. 69 (2), 303-309.

Branzoli, F., Ercan, E., Webb, A., Ronen, I., 2014. The interaction between apparent diffusion coefficients and transverse relaxation rates of human brain metabolites and water studied by diffusion-weighted spectroscopy at 7 T. Nmr Biomed. 27 (5), 495-506.

Branzoli, F., et al., 2016. Differentiating between axonal damage and demyelination in healthy aging by combining diffusion-tensor imaging and diffusion-weighted spectroscopy in the human corpus callosum at 7 T. Neurobiol. Aging 47, 210-217.

Budde, M.D., Frank, J.A., 2010. Neurite beading is sufficient to decrease the apparent diffusion coefficient after ischemic stroke. Proc. Natl. Acad. Sci. U. S. A. 107 (32), $14472-14477$.

Callaghan, P.T., 1991. Principles of Nuclear Magnetic Resonance Microscopy. Clarendon Press ; Oxford University Press.
Callaghan, P.T., 1995. Pulsed-gradient spin-echo Nmr for planar, cylindrical, and spherical pores under conditions of wall relaxation. J. Magn. Reson Ser. A 113 (1) 53-59.

Callaghan, P.T., Coy, A., Macgowan, D., Packer, K.J., Zelaya, F.O., 1991. Diffraction-like effects in Nmr diffusion studies of fluids in porous solids. Nature 351 (6326), 467-469.

Cao, P., Wu, E.X., 2015. Accelerating phase-encoded proton MR spectroscopic imaging by compressed sensing. J. Magn. Reson Imaging 41 (2), 487-495.

Cao, P., Wu, E.X., 2017. In vivo diffusion MRS investigation of non-water molecules in biological tissues. Nmr Biomed. 30 (3).

Cao, P., et al., 2016. Accelerated high-bandwidth MR spectroscopic imaging using compressed sensing. Magn. Reson Med. 76 (2), 369-379.

Cerdan, S., Kunnecke, B., Seelig, J., 1990. Cerebral metabolism of [1,2-13C2]acetate as detected by in vivo and in vitro 13C NMR. J. Biol. Chem. 265 (22), 12916-12926.

Chang, L.C., Koay, C.G., Pierpaoli, C., Basser, P.J., 2007. Variance of estimated DTIderived parameters via first-order perturbation methods. Magn. Reson Med. 57 (1), $141-149$.

Choi, J.K., Dedeoglu, A., Jenkins, B.G., 2007. Application of MRS to mouse models of neurodegenerative illness. Nmr Biomed. 20 (3), 216-237.

Chvatal, A., Anderova, M., Kirchhoff, F., 2007. Three-dimensional confocal morphometry - a new approach for studying dynamic changes in cell morphology in brain slices. J. Anat. 210 (6), 671-683.

Cohen, Y., Assaf, Y., 2002. High b-value q-space analyzed diffusion-weighted MRS and MRI in neuronal tissues - a technical review. Nmr Biomed. 15 (7-8), 516-542.

Cory, D.G., Garroway, A.N., Miller, J.B., 1990. Applications of spin transport as a probe of local geometry. Abstr. Pap. Am. Chem. S 199, 105-Poly.

Delacourte, A., Buee, L., 2000. Tau pathology: a marker of neurodegenerative disorders. Curr. Opin. Neurol. 13 (4), 371-376.

Dijkhuizen, R.M., de Graaf, R.A., Tulleken, K.A., Nicolay, K., 1999. Changes in the diffusion of water and intracellular metabolites after excitotoxic injury and global ischemia in neonatal rat brain. J. Cereb. Blood Flow. Metab. 19 (3), 341-349.

Dreher, W., Busch, E., Leibfritz, D., 2001. Changes in apparent diffusion coefficients of metabolites in rat brain after middle cerebral artery occlusion measured by proton magnetic resonance spectroscopy. Magn. Reson Med. 45 (3), 383-389.

Drobnjak, I., Zhang, H., Hall, M.G., Alexander, D.C., 2011. The matrix formalism for generalised gradients with time-varying orientation in diffusion NMR. J. Magn. Reson 210 (1), 151-157.

Du, F., et al., 2013. Myelin and axon abnormalities in schizophrenia measured with magnetic resonance imaging techniques. Biol. Psychiatry 74 (6), 451-457.

Duarte, J.M., Lei, H., Mlynarik, V., Gruetter, R., 2012. The neurochemical profile quantified by in vivo 1H NMR spectroscopy. Neuroimage 61 (2), 342-362.

Ellegood, J., Hanstock, C.C., Beaulieu, C., 2005. Trace apparent diffusion coefficients of metabolites in human brain using diffusion weighted magnetic resonance spectroscopy. Magn. Reson Med. 53 (5), 1025-1032.

Ellegood, J., Hanstock, C.C., Beaulieu, C., 2006. Diffusion tensor spectroscopy (DTS) of human brain. Magn. Reson Med. 55 (1), 1-8.

Ellegood, J., McKay, R.T., Hanstock, C.C., Beaulieu, C., 2007. Anisotropic diffusion of metabolites in peripheral nerve using diffusion weighted magnetic resonance spectroscopy at ultra-high field. J. Magn. Reson 184 (1), 20-28.

Ellegood, J., Hanstock, C.C., Beaulieu, C., 2011. Considerations for measuring the fractional anisotropy of metabolites with diffusion tensor spectroscopy. Nmr Biomed. 24 (3), 270-280.

Ercan, A.E., Techawiboonwong, A., Versluis, M.J., Webb, A.G., Ronen, I., 2015. Diffusionweighted chemical shift imaging of human brain metabolites at 7T. Magn. Reson Med. 73 (6), 2053-2061.

Ercan, E., et al., 2016. Glial and axonal changes in systemic lupus erythematosus measured with diffusion of intracellular metabolites. Brain 139 (Pt 5), 1447-1457.

Feinberg, D.A., Setsompop, K., 2013. Ultra-fast MRI of the human brain with simultaneous multi-slice imaging. J. Magn. Reson 229, 90-100.

Finsterbusch, J., 2010. Multiple-Wave-Vector diffusion-weighted NMR. Annu. Rep. Nmr Spectro 72, 225-299.

Fotso, K., et al., 2017. Diffusion tensor spectroscopic imaging of the human brain in children and adults. Magn. Reson Med. 78 (4), 1246-1256.

Frahm, J., Merboldt, K.D., Hanicke, W., 1987. Localized proton spectroscopy using stimulated echoes. J. Magn. Reson 72, 502-508.

Garwood, M., DelaBarre, L., 2001. The return of the frequency sweep: designing adiabatic pulses for contemporary NMR. J. Magn. Reson 153 (2), 155-177.

Gill, S.S., et al., 1989. Brain metabolites as 1H NMR markers of neuronal and glial disorders. Nmr Biomed. 2 (5-6), 196-200.

de Graaf, R.A., Braun, K.P., Nicolay, K., 2001. Single-shot diffusion trace (1)H NMR spectroscopy. Magn. Reson Med. 45 (5), 741-748.

de Graaf, R.A., et al., 2006. High magnetic field water and metabolite proton T1 and T2 relaxation in rat brain in vivo. Magn. Reson Med. 56 (2), 386-394.

Grebenkov, D.S., 2007. NMR survey of reflected Brownian motion. Rev. Mod. Phys. 79 (3), 1077-1137.

Grundke-Iqbal, I., et al., 1986. Abnormal phosphorylation of the microtubule-associated protein tau (tau) in Alzheimer cytoskeletal pathology. Proc. Natl. Acad. Sci. U. S. A. 83 (13), 4913-4917.

Hahn, E.L., 1950. Spin echoes. Phys. Rev. 77 (5), 746-746.

Hakumaki, J.M., Pirttila, T.R., Kauppinen, R.A., 2000. Reduction in water and metabolite apparent diffusion coefficients during energy failure involves cation-dependent mechanisms. A proton nuclear magnetic resonance study of rat cortical brain slices. J. Cereb. Blood Flow. Metab. 20 (2), 405-411.

Harada, M., et al., 2002. Diffusion-weighted in vivo localized proton MR spectroscopy of human cerebral ischemia and tumor. Nmr Biomed. 15 (1), 69-74. 
Ianus, A., Alexander, D.C., Drobnjak, I., 2016. Microstructure imaging sequence simulation toolbox. Lect. Notes Comput. Sc 9968, 34-44.

Jara, H., Wehrli, F.W., 1994. Determination of background gradients with diffusion MR imaging. J. Magn. Reson Imaging 4 (6), 787-797.

Jespersen, S.N., Kroenke, C.D., Ostergaard, L., Ackerman, J.J., Yablonskiy, D.A., 2007. Modeling dendrite density from magnetic resonance diffusion measurements. Neuroimage 34 (4), 1473-1486.

Jespersen, S.N., Lundell, H., Sonderby, C.K., Dyrby, T.B., 2013. Orientationally invariant metrics of apparent compartment eccentricity from double pulsed field gradient diffusion experiments. Nmr Biomed. 26 (12), 1647-1662.

Kan, H.E., et al., 2012. Differences in apparent diffusion coefficients of brain metabolites between grey and white matter in the human brain measured at 7 T. Magn. Reson Med. 67 (5), 1203-1209.

Keeler, J., 2011. Understanding NMR Spectroscopy. John Wiley \& Sons.

Kiselev, V.G., 2017. Fundamentals of diffusion MRI physics. Nmr Biomed. 30 (3).

Koch, M.A., Finsterbusch, J., 2011. Towards compartment size estimation in vivo based on double wave vector diffusion weighting. Nmr Biomed. 24 (10), 1422-1432.

Kroenke, C.D., Ackerman, J.J., Neil, J.J., 2003. Magnetic resonance measurement of tetramethylammonium diffusion in rat brain: comparison of magnetic resonance and ionophoresis in vivo diffusion measurements. Magn. Reson Med. 50 (4), 717-726.

Kroenke, C.D., Ackerman, J.J., Yablonskiy, D.A., 2004. On the nature of the NAA diffusion attenuated MR signal in the central nervous system. Magn. Reson Med. 52 (5), $1052-1059$.

Kunnecke, B., Cerdan, S., Seelig, J., 1993. Cerebral metabolism of [1,2-13C2]glucose and [U-13C4]3-hydroxybutyrate in rat brain as detected by 13C NMR spectroscopy. Nmr Biomed. 6 (4), 264-277.

Lampinen, B., et al., 2017. Neurite density imaging versus imaging of microscopic anisotropy in diffusion MRI: a model comparison using spherical tensor encoding. Neuroimage 147, 517-531.

Latour, L.L., Svoboda, K., Mitra, P.P., Sotak, C.H., 1994. Time-dependent diffusion of water in a biological model system. P Natl. Acad. Sci. U. S. A. 91 (4), 1229-1233.

Lawrenz, M., Finsterbusch, J., 2013. Double-wave-vector diffusion-weighted imaging reveals microscopic diffusion anisotropy in the living human brain. Magn. Reson Med. 69 (4), 1072-1082.

Lawrenz, M., Brassen, S., Finsterbusch, J., 2015. Microscopic diffusion anisotropy in the human brain: reproducibility, normal values, and comparison with the fractional anisotropy. Neuroimage 109, 283-297.

Lee, P.L., Gonzalez, R.G., 2000. Magnetic resonance spectroscopy of brain tumors. Curr. Opin. Oncol. 12 (3), 199-204.

Ligneul, C., Valette, J., 2017. Probing metabolite diffusion at ultra-short time scales in the mouse brain using optimized oscillating gradients and "short"-echo-time diffusionweighted MRS. Nmr Biomed. 30 (1).

Ligneul, C., Palombo, M., Valette, J., 2017. Metabolite diffusion up to very high b in the mouse brain in vivo: revisiting the potential correlation between relaxation and diffusion properties. Magn. Reson Med. 77 (4), 1390-1398.

Linse, P., Soderman, O., 1995. The validity of the short-gradient-pulse approximation in Nmr-studies of restricted diffusion - simulations of molecules diffusing between planes, in cylinders and spheres. J. Magn. Reson Ser. A 116 (1), 77-86.

Lori, N.F., et al., 2002. Diffusion tensor fiber tracking of human brain connectivity: acquisition methods, reliability analysis and biological results. Nmr Biomed. 15 (7-8), 494-515.

Lundell, H., Ingo, C., Dyrby, T.B., Ronen, I., 2017. Accurate estimation of intra-axonal diffusivity and anisotropy of NAA in humans at 7T. Proc. Intl. Soc. Mag. Reson. Med. 25.

Lundgaard, I., Osorio, M.J., Kress, B.T., Sanggaard, S., Nedergaard, M., 2014. White matter astrocytes in health and disease. Neuroscience 276, 161-173.

MacKay, A., et al., 1994. In vivo visualization of myelin water in brain by magnetic resonance, Magn. Reson Med. 31 (6), 673-677.

Mangia, S., et al., 2009. Metabolic and hemodynamic events after changes in neuronal activity: current hypotheses, theoretical predictions and in vivo NMR experimental findings. J. Cereb. Blood Flow. Metab. 29 (3), 441-463.

Marchadour, C., Brouillet, E., Hantraye, P., Lebon, V., Valette, J., 2012. Anomalous diffusion of brain metabolites evidenced by diffusion-weighted magnetic resonance spectroscopy in vivo. J. Cereb. Blood Flow. Metab. 32 (12), 2153-2160.

Merboldt, K.D., Horstermann, D., Hanicke, W., Bruhn, H., Frahm, J., 1993. Molecular selfdiffusion of intracellular metabolites in rat brain in vivo investigated by localized proton NMR diffusion spectroscopy. Magn. Reson Med. 29 (1), 125-129.

Mierisova, S., et al., 1998. New approach for quantitation of short echo time in vivo $1 \mathrm{H}$ MR spectra of brain using AMARES. Nmr Biomed. 11 (1), 32-39.

Mitra, P.P., 1995. Multiple wave-vector extensions of the Nmr pulsed-field-gradient spinecho diffusion measurement. Phys. Rev. B 51 (21), 15074-15078.

Mitra, P.P., Sen, P.N., Schwartz, L.M., 1993. Short-time behavior of the diffusioncoefficient as a geometrical probe of porous-media. Phys. Rev. B 47 (14), 8565-8574.

Molloy, G.R., Wilson, C.D., Benfield, P., de Vellis, J., Kumar, S., 1992. Rat brain creatine kinase messenger RNA levels are high in primary cultures of brain astrocytes and oligodendrocytes and low in neurons. J. Neurochem. 59 (5), 1925-1932.

Moonen, C.T., van Zijl, P.C., Le Bihan, D., DesPres, D., 1990. In vivo NMR diffusion spectroscopy: 31P application to phosphorus metabolites in muscle. Magn. Reson Med. 13 (3), 467-477.

Mori, S., van Zijl, P.C.M., 2002. Fiber tracking: principles and strategies - a technical review. Nmr Biomed. 15 (7-8), 468-480.

Morris, K.F., Johnson, C.S., 1993. Resolution of discrete and continuous molecular-size distributions by means of diffusion-ordered 2d Nmr-spectroscopy. J. Am. Chem. Soc. 115 (10), 4291-4299.
Moseley, M.E., et al., 1990. Diffusion-weighted MR imaging of acute stroke: correlation with T2-weighted and magnetic susceptibility-enhanced MR imaging in cats. AJNR Am. J. Neuroradiol. 11 (3), 423-429.

Najac, C., et al., 2014. Intracellular metabolites in the primate brain are primarily localized in long fibers rather than in cell bodies, as shown by diffusion-weighted magnetic resonance spectroscopy. Neuroimage 90, 374-380.

Najac, C., Branzoli, F., Ronen, I., Valette, J., 2016. Brain intracellular metabolites are freely diffusing along cell fibers in grey and white matter, as measured by diffusionweighted MR spectroscopy in the human brain at 7 T. Brain Struct. Funct. 221 (3), 1245-1254.

Neeman, M., Freyer, J.P., Sillerud, L.O., 1991. A simple method for obtaining cross-termfree images for diffusion anisotropy studies in NMR microimaging. Magn. Reson Med. 21 (1), 138-143.

Nicolay, K., van der Toorn, A., Dijkhuizen, R.M., 1995. In vivo diffusion spectroscopy. An overview. Nmr Biomed. 8 (7-8), 365-374.

Nicolay, K., Braun, K.P., Graaf, R.A., Dijkhuizen, R.M., Kruiskamp, M.J., 2001. Diffusion NMR spectroscopy. Nmr Biomed. 14 (2), 94-111.

Nicoli, F., et al., 2003. Metabolic counterpart of decreased apparent diffusion coefficient during hyperacute ischemic stroke - a brain proton magnetic resonance spectroscopic imaging study. Stroke 34 (7), E82-E87.

Nilsson, M., et al., 2013. Noninvasive mapping of water diffusional exchange in the human brain using filter-exchange imaging. Magn. Reson Med. 69 (6), 1573-1581.

Nilsson, M., Lasic, S., Drobnjak, I., Topgaard, D., Westin, C.F., 2017. Resolution limit of cylinder diameter estimation by diffusion MRI: the impact of gradient waveform and orientation dispersion. Nmr Biomed. 30 (7), e3711.

Nordengen, K., Heuser, C., Rinholm, J.E., Matalon, R., Gundersen, V., 2015. Localisation of N-acetylaspartate in oligodendrocytes/myelin. Brain Struct. Funct. 220 (2), 899-917.

Novikov, D.S., Kiselev, V.G., 2011. Surface-to-volume ratio with oscillating gradients. J. Magn. Reson 210 (1), 141-145.

Novikov, D.S., Jensen, J.H., Helpern, J.A., Fieremans, E., 2014. Revealing mesoscopic structural universality with diffusion. P Natl. Acad. Sci. U. S. A. 111 (14), 5088-5093.

Oberheim, N.A., et al., 2009. Uniquely hominid features of adult human astrocytes. J. Neurosci. 29 (10), 3276-3287.

Oeltzschner, G., et al., 2017. Dual-volume excitation and parallel reconstruction for Jdifference-edited MR spectroscopy. Magn. Reson Med. 77 (1), 16-22.

Ordidge, R.J., Connelly, A., Lohman, J.A.B., 1986. Image-selected invivo spectroscopy (isis) - a new technique for spatially selective Nmr-spectroscopy. J. Magn. Reson 66 (2), 283-294.

Ozarslan, E., Shemesh, N., Basser, P.J., 2009. A general framework to quantify the effect of restricted diffusion on the NMR signal with applications to double pulsed field gradient NMR experiments. J. Chem. Phys. 130 (10).

Palombo, M., et al., 2016. New paradigm to assess brain cell morphology by diffusionweighted MR spectroscopy in vivo. Proc. Natl. Acad. Sci. U. S. A. 113 (24), 6671-6676.

Palombo, M., Ligneul, C., Valette, J., 2017. Modeling diffusion of intracellular metabolites in the mouse brain up to very high diffusion-weighting: diffusion in long fibers (almost) accounts for non-monoexponential attenuation. Magn. Reson Med. 77 (1) 343-350.

Petroff, O.A., Pleban, L., Prichard, J.W., 1993. Metabolic assessment of a neuron-enriched fraction of rat cerebrum using high-resolution 1H and 13C NMR spectroscopy. Magn. Reson Med. 30 (5), 559-567.

Pfeuffer, J., Tkac, I., Gruetter, R., 2000. Extracellular-intracellular distribution of glucose and lactate in the rat brain assessed noninvasively by diffusion-weighted $1 \mathrm{H}$ nuclear magnetic resonance spectroscopy in vivo. J. Cereb. Blood Flow. Metab. 20 (4), $736-746$.

Posse, S., Cuenod, C.A., Le Bihan, D., 1993. Human brain: proton diffusion MR spectroscopy. Radiology 188 (3), 719-725.

Price, W.S., 2009. NMR Studies of Translational Motion: Principles and Applications. Cambridge University Press.

Price, W.S., Chapman, B.E., Cornell, B.A., Kuchel, P.W., 1989. Translational diffusion of Glycine in erythrocytes measured at high-resolution with pulsed field gradients. J. Magn. Reson 83 (1), 160-166.

Provencher, S.W., 1993. Estimation of metabolite concentrations from localized in vivo proton NMR spectra. Magn. Reson Med. 30 (6), 672-679.

Ronen, I., Valette, J., 2015. Diffusion-weighted magnetic resonance spectroscopy. eMagRes 4, 733-750.

Ronen, I., Ercan, E., Webb, A., 2013. Axonal and glial microstructural information obtained with diffusion-weighted magnetic resonance spectroscopy at 7T. Front. Integr. Neurosci. 7, 13.

Ronen, I., et al., 2014. Microstructural organization of axons in the human corpus callosum quantified by diffusion-weighted magnetic resonance spectroscopy of Nacetylaspartate and post-mortem histology. Brain Struct. Funct. 219 (5), 1773-1785.

Rothman, D.L., et al., 1999. In vivo nuclear magnetic resonance spectroscopy studies of the relationship between the glutamate-glutamine neurotransmitter cycle and functional neuroenergetics. Philos. Trans. R. Soc. Lond B Biol. Sci. 354 (1387), 1165-1177.

Schlaug, G., Siewert, B., Benfield, A., Edelman, R.R., Warach, S., 1997. Time course of the apparent diffusion coefficient (ADC) abnormality in human stroke. Neurology 49 (1), $113-119$.

Sen, P.N., 2004. Time-dependent diffusion coefficient as a probe of geometry. Concept Magn. Reson A 23a (1), 1-21.

Shemesh, N., Cohen, Y., 2008. The effect of experimental parameters on the signal decay in double-PGSE experiments: negative diffractions and enhancement of structural information. J. Magn. Reson 195 (2), 153-161. 
Shemesh, N., Cohen, Y., 2011. Overcoming apparent Susceptibility-Induced Anisotropy (aSIA) by bipolar double-Pulsed-Field-Gradient NMR. J. Magn. Reson 212 (2), 362-369.

Shemesh, N., Ozarslan, E., Adiri, T., Basser, P.J., Cohen, Y., 2010. Noninvasive bipolar double-pulsed-field-gradient NMR reveals signatures for pore size and shape in polydisperse, randomly oriented, inhomogeneous porous media. J. Chem. Phys. 133 (4).

Shemesh, N., Adiri, T., Cohen, Y., 2011. Probing microscopic architecture of opaque heterogeneous systems using double-pulsed-field-gradient NMR. J. Am. Chem. Soc. 133 (15), 6028-6035.

Shemesh, N., Ozarslan, E., Basser, P.J., Cohen, Y., 2012. Accurate noninvasive measurement of cell size and compartment shape anisotropy in yeast cells using double-pulsed field gradient MR. Nmr Biomed. 25 (2), 236-246.

Shemesh, N., Rosenberg, J.T., Dumez, J.N., Grant, S.C., Frydman, L., 2014a. Metabolic T1 dynamics and longitudinal relaxation enhancement in vivo at ultrahigh magnetic fields on ischemia. J. Cereb. Blood Flow. Metab. 34 (11), 1810-1817.

Shemesh, N., et al., 2014b. Metabolic properties in stroked rats revealed by relaxationenhanced magnetic resonance spectroscopy at ultrahigh fields. Nat. Commun. 5.

Shemesh, N., Rosenberg, J.T., Dumez, J.-N., Frydman, L., Grant, S.C., 2015. Resolving cellular specific microarchitectures using double pulsed field gradient weighted, relaxation-enhanced magnetic resonance spectroscopy. Proc. Intl. Soc. Mag. Reson. Med. 23.

Shemesh, N., et al., 2016. Conventions and nomenclature for double diffusion encoding NMR and MRI. Magn. Reson Med. 75 (1), 82-87.

Simmons, M.L., Frondoza, C.G., Coyle, J.T., 1991. Immunocytochemical localization of Nacetyl-aspartate with monoclonal antibodies. Neuroscience 45 (1), 37-45.

Soderman, O., Jonsson, B., 1995. Restricted diffusion in cylindrical geometry. J. Magn. Reson Ser. A 117 (1), 94-97.

Stejskal, E.O., Tanner, J.E., 1965. Spin diffusion measurements: spin echoes in the presence of a time-dependent field gradient. J. Chem. Phys. 42 (1), 288-+.

Stepisnik, J., 1993. Time-dependent self-diffusion by Nmr spin-echo. Phys. B-Condensed Matter 183 (4), 343-350.

Tkac, I., Oz, G., Adriany, G., Ugurbil, K., Gruetter, R., 2009. In vivo 1H NMR spectroscopy of the human brain at high magnetic fields: metabolite quantification at 4T vs. 7T. Magn. Reson Med. 62 (4), 868-879.

van der Toorn, A., Dijkhuizen, R.M., Tulleken, C.A., Nicolay, K., 1996. Diffusion of metabolites in normal and ischemic rat brain measured by localized 1H MRS. Magn. Reson Med. 36 (6), 914-922.

Upadhyay, J., Hallock, K., Erb, K., Kim, D.S., Ronen, I., 2007. Diffusion properties of NAA in human corpus callosum as studied with diffusion tensor spectroscopy. Magn. Reson Med. 58 (5), 1045-1053.

Upadhyay, J., Hallock, K., Ducros, M., Kim, D.S., Ronen, I., 2008. Diffusion tensor spectroscopy and imaging of the arcuate fasciculus. Neuroimage 39 (1), 1-9.
Valette, J., et al., 2005. Optimized diffusion-weighted spectroscopy for measuring brain glutamate apparent diffusion coefficient on a whole-body MR system. Nmr Biomed. 18 (8), 527-533.

Valette, J., et al., 2012. A new sequence for single-shot diffusion-weighted NMR spectroscopy by the trace of the diffusion tensor. Magn. Reson Med. 68 (6), 1705-1712.

Vangelderen, P., et al., 1994. Water diffusion and acute stroke. Magn. Reson Med. 31 (2), $154-163$.

Vanhamme, L., Van Huffel, S., Van Hecke, P., van Ormondt, D., 1999. Time-domain quantification of series of biomedical magnetic resonance spectroscopy signals. J. Magn. Reson 140 (1), 120-130.

Vanzijl, P.C.M., et al., 1991. Complete separation of intracellular and extracellular information in Nmr-spectra of perfused cells by diffusion-weighted spectroscopy P Natl. Acad. Sci. U. S. A. 88 (8), 3228-3232.

Verkhratsky, A., Parpura, V., Pekna, M., Pekny, M., Sofroniew, M., 2014. Glia in the pathogenesis of neurodegenerative diseases. Biochem. Soc. Trans. 42 (5), 1291-1301.

Wick, M., Nagatomo, Y., Prielmeier, F., Frahm, J., 1995. Alteration of intracellular metabolite diffusion in rat brain in vivo during ischemia and reperfusion. Stroke 26 (10), 1930-1933 discussion 1934.

Wijnen, J.P., et al., 2014. 1D diffusion weighted magnetic resonance spectroscopy along the arcuate fasiculus in the human brain at 7T. Int. Soc. Magnetic Reson. Med. p. 694.

Wilson, M., Reynolds, G., Kauppinen, R.A., Arvanitis, T.N., Peet, A.C., 2011. A constrained least-squares approach to the automated quantitation of in vivo (1)H magnetic resonance spectroscopy data. Magn. Reson Med. 65 (1), 1-12.

Wood, E.T., et al., 2012. Investigating axonal damage in multiple sclerosis by diffusion tensor spectroscopy. J. Neurosci. 32 (19), 6665-6669.

Wood, E.T., et al., 2017. Longitudinal MR spectroscopy of neurodegeneration in multiple sclerosis with diffusion of the intra-axonal constituent $\mathrm{N}$-acetylaspartate. Neuroimage Clin. 15, 780-788.

Yablonskiy, D.A., Sukstanskii, A.L., 2010. Theoretical models of the diffusion weighted MR signal. Nmr Biomed. 23 (7), 661-681.

Yoshiura, T., Wu, O., Zaheer, A., Reese, T.G., Sorensen, A.G., 2001. Highly diffusionsensitized MRI of brain: dissociation of gray and white matter. Magn. Reson Med. 45 (5), 734-740.

Zhang, H., Schneider, T., Wheeler-Kingshott, C.A., Alexander, D.C., 2012. NODDI: practical in vivo neurite orientation dispersion and density imaging of the human brain. Neuroimage 61 (4), 1000-1016.

Zheng, G., Price, W.S., 2007. Suppression of background gradients in (B-0 gradient-based) NMR diffusion experiments. Concept Magn. Reson A 30a (5), 261-277.

Zheng, D.D., Liu, Z.H., Fang, J., Wang, X.Y., Zhang, J., 2012. The effect of age and cerebral ischemia on diffusion-weighted proton MR spectroscopy of the human brain. AJNR Am. J. Neuroradiol. 33 (3), 563-568. 